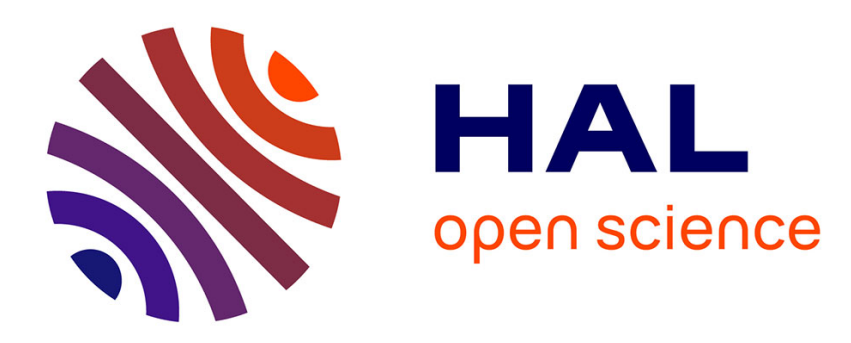

\title{
A Survey of Karin Cluster Asteroids with the Spitzer Space Telescope
}

\author{
Alan W. Harris, Michael Mueller, Carey M. Lisse, Andrew F. Cheng
}

\section{To cite this version:}

Alan W. Harris, Michael Mueller, Carey M. Lisse, Andrew F. Cheng. A Survey of Karin Cluster Asteroids with the Spitzer Space Telescope. Icarus, 2008, 199 (1), pp.86. 10.1016/j.icarus.2008.09.004 . hal-00499096

\section{HAL Id: hal-00499096 https://hal.science/hal-00499096}

Submitted on 9 Jul 2010

HAL is a multi-disciplinary open access archive for the deposit and dissemination of scientific research documents, whether they are published or not. The documents may come from teaching and research institutions in France or abroad, or from public or private research centers.
L'archive ouverte pluridisciplinaire HAL, est destinée au dépôt et à la diffusion de documents scientifiques de niveau recherche, publiés ou non, émanant des établissements d'enseignement et de recherche français ou étrangers, des laboratoires publics ou privés. 


\section{Accepted Manuscript}

A Survey of Karin Cluster Asteroids with the Spitzer Space Telescope

Alan W. Harris, Michael Mueller, Carey M. Lisse, Andrew F. Cheng

PII:

S0019-1035(08)00323-0

DOI: $\quad$ 10.1016/j.icarus.2008.09.004

Reference: $\quad$ YICAR 8763

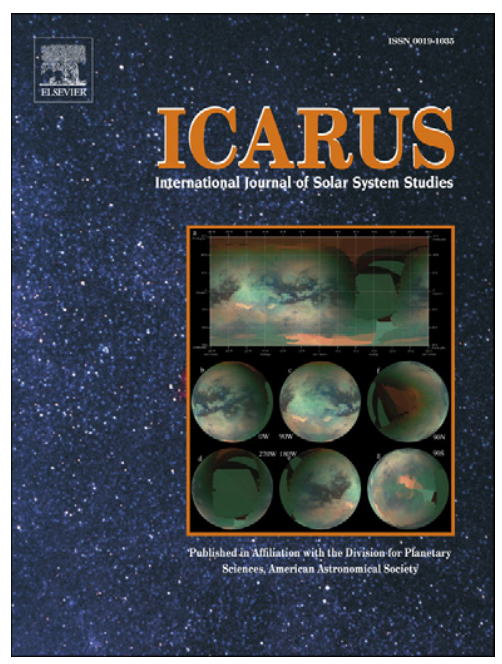

To appear in: Icarus

Received date: 6 August 2008

Revised date: 25 September 2008

Accepted date: 26 September 2008

Please cite this article as: A.W. Harris, M. Mueller, C.M. Lisse, A.F. Cheng, A Survey of Karin Cluster Asteroids with the Spitzer Space Telescope, Icarus (2008), doi: 10.1016/j.icarus.2008.09.004

This is a PDF file of an unedited manuscript that has been accepted for publication. As a service to our customers we are providing this early version of the manuscript. The manuscript will undergo copyediting, typesetting, and review of the resulting proof before it is published in its final form. Please note that during the production process errors may be discovered which could affect the content, and all legal disclaimers that apply to the journal pertain. 


\title{
A Survey of Karin Cluster Asteroids with the Spitzer Space Telescope
}

\author{
Alan W. Harris \\ DLR Institute of Planetary Research, Rutherfordstrasse 2, 12489 Berlin, Germany \\ E-mail: alan.harris@dlr.de \\ Tel: +49 3067055324 \\ Fax: +493067055303
}

\section{Michael Mueller}

DLR Institute of Planetary Research, Rutherfordstrasse 2, 12489 Berlin, Germany

(current address: University of Arizona, Steward Observatory, 933 N Cherry Ave, Tucson AZ 85721, USA)

\section{Carey M. Lisse, Andrew F. Cheng}

Planetary Exploration Group, Space Department, Johns Hopkins University Applied Physics Laboratory, 11100 Johns

Hopkins Rd, Laurel, MD 20723, USA

Submitted to Icarus

\section{$\underline{\text { Revised version, } 2008 \text { September } 25}$}

No. of manuscript pages: 38 (excl. figures)

No. of figures: 6

No. of tables: 7 
Running head: Spitzer observations of Karin Cluster Asteroids

Editorial correspondence to:

Alan W. Harris

DLR Institute of Planetary Research

Rutherfordstrasse 2

12489 Berlin

Germany

E-mail: alan.harris@dlr.de 


\section{Abstract}

The Karin cluster is one of the youngest known families of main-belt asteroids, dating back to a collisional event only $5.8 \pm 0.2$ Myr ago. Using the Spitzer Space Telescope we have photometrically sampled the thermal continua $(3.5-22 \mu \mathrm{m})$ of 17 Karin cluster asteroids of different sizes, down to the smallest members discovered so far, in order to make the first direct measurements of their sizes and albedos and study the physical properties of their surfaces. Our targets are also amongst the smallest main-belt asteroids observed to date in the mid-infrared. The derived diameters range from $17.3 \mathrm{~km}$ for 832 Karin to $1.5 \mathrm{~km}$ for 75176 , with typical uncertainties of $10 \%$. The mean albedo is $p_{\mathrm{v}}=0.215 \pm 0.015$, compared to $0.20 \pm$ 0.07 for 832 Karin itself (for $H=11.2 \pm 0.3$ ), consistent with the view that the Karin asteroids are closely related physically as well as dynamically. The albedo distribution $\left(0.12 \leq p_{\mathrm{v}} \leq 0.32\right)$ is consistent with the range associated with S-type asteroids but the variation from one object to another appears to be significant. Contrary to the case for near-Earth asteroids, our data show no evidence of an albedo dependence on size. However, the mean albedo is lower than expected for young, fresh "S-type" surfaces, suggesting that space weathering can darken main-belt asteroid surfaces on very short timescales. Our data are also suggestive of a connection between surface roughness and albedo, which may reflect rejuvenation of weathered surfaces by impact gardening. While the available data allow only estimates of lower limits for thermal inertia, we find no evidence for the relatively high values of thermal inertia reported for some similarly-sized near-Earth asteroids. Our results constitute the first observational confirmation of the legitimacy of assumptions made in recent modeling of the formation of the Karin cluster via a single catastrophic collision 5.8 $\pm 0.2 \mathrm{Myr}$ ago.

Keywords: Asteroids, Infrared Observations, Photometry, Spectrophotometry 


\section{Introduction}

The Karin cluster, named after its largest member, (832) Karin, is believed to have been formed $5.8 \pm 0.2 \mathrm{Myr}$ ago in a catastrophic collision between two asteroids in the main belt (Nesvorný et al., 2002). This cluster is of great interest owing to the possibility that the physical properties of its members may preserve unique information about asteroid fragmentation as well as surface processes on small asteroids, which include regolith formation and modification of albedo and spectral properties by space weathering.

The Karin cluster can be identified in so-called proper element space (the proper orbital elements are approximate constants of the motion after considering perturbations from the planets) as an unusually tight grouping of objects, even when compared with the groupings that define the classical, well-known asteroid families which are believed typically to be much older (at least hundreds of millions of years) and to have dispersed dynamically since formation. The Karin cluster is found very close to the center of the Koronis family of which it is a sub-family; so detailed orbital integrations are required to distinguish Karin cluster members from background objects in the Koronis family (Nesvorný and Bottke, 2004). The extremely young reported age of the Karin cluster provides a unique opportunity to study the fragmentation of asteroids. Because of the short collisional lifetimes of small asteroids in the main-belt, the young cluster age minimizes further fragmentation after the original event and limits the orbital dispersal of cluster members. Studies of the fragmentation size distribution are important because the effects of collisions shape the total asteroid size distribution (Davis et al., 2002). Collisions cause the destruction and erosion of asteroids as well as the injection of new bodies. Recent modeling (e.g., Cheng, 2004) suggests that features in the overall main-belt asteroid (MBA) size distribution, which is not a simple power law, reflect size-dependent 
fragmentation physics and internal structure of asteroids, but our understanding of asteroid collisional evolution is highly uncertain and incomplete. There is strong evidence that the observed infrared excesses observed in the spectra of some main-sequence stars are due to dust created by stochastic collisional fragmentation of large $(>10 \mathrm{~km})$ asteroids (e.g. Lisse et al., 2007). The understanding of asteroid collisions and family formation is thus of importance for understanding planetary system development in general. The formation of the Karin family is one of the most recent occurrences of such an event in our Solar System. Nesvorný et al. (2006) have modeled the formation of the Karin cluster and conclude that the cluster was formed by a highly catastrophic disruption of a largely unfractured asteroid with a diameter of about $33 \mathrm{~km}$. Our work enables crucial assumptions made by Nesvorný et al. regarding the albedo distribution and sizes of the Karin cluster members to be tested observationally for the first time.

\section{Observations and data reduction}

A total of 17 Karin-cluster asteroids were observed using the Spitzer Space Telescope (hereafter Spitzer; Werner et al., 2004) between August 2005 and July 2007 (under Spitzer Cycle 2 General Observer Program \#20158). Each target was imaged at six wavelengths, using the Infrared Array Camera, IRAC (Fazio et al., 2004) and the Infrared Spectrograph, IRS (Houck et al., 2004) in peak-up imaging (PUI) mode. IRAC provides four filter passbands (referred to as channels 1 - 4 in the following) with central wavelengths of 3.550, 4.493, 5.731, and $7.872 \mu \mathrm{m}$, respectively. IRS PUI provides two passbands centered at 15.8 and $22.3 \mu \mathrm{m}$. For more details the reader is referred to the instrument-specific data handbooks at http://ssc.spitzer.caltech.edu/irac/dh/ and http://ssc.spitzer.caltech.edu/irs/dh/. 
IRAC was used in standard imaging mode. Channels 1 and 3 share a common field-of-view (FOV) using a beam splitter, as do channels 2 and 4. In most observations, channels 2 and 4 were on target for the first half of the total integration time, followed by channels 1 and 3 for the second half. At each position a standard dither pattern was executed. Observations of faint targets are subject to confusion with background sources, which can be mitigated against by taking advantage of the motion of our targets. Observations of four targets that were anticipated to be weak were designed to maximize the temporal baseline for each channel pair by having the two FOVs "take turns" on the target (see the discussion of IRAC observing strategy by Mueller, 2007): with integrations in channels $2+4$ on target denoted as "A", and those in channels $1+3$ on target denoted as "B", the observing patterns were ABAB for 55124 and 76686; and ABABABAB for 75176 and 76019.

Since only one Spitzer instrument is powered on at any time, the observations with the two instruments were performed at different epochs, days or weeks apart, introducing systematic uncertainties due to rotational variation of the integrated surface albedos and projected crosssectional areas. For all observations, the respective FOV was dithered five or more times around the nominal target position using standard Spitzer dither patterns with step sizes of a few tens of arc seconds. The targets remained on chip at all dither positions. See Table 1 for a list of the target asteroids, observation times, and relevant parameters. Table 2 lists absolute magnitudes ( $H$ values) for the targets and those adopted for this study.

The Spitzer Science Center's automated IRAC and PUI data reduction pipelines provided 'basic calibrated data' (BCD) frames, corrected for most instrument artifacts (including dark current and flat-field effects), with an absolute flux calibration applied. We used the software package MOPEX (see Makovoz and Khan, 2005) to co-add BCD images in the asteroid rest frame, after matching their background levels and rejecting non-co-moving sources such as 
stellar background sources or cosmic ray hits. All targets were clearly visible at their nominal positions. Occasionally, nearby background sources (which in the case of deep IRAC observations trailed up to several tens of pixels, at a pixel scale of 1.8") or image artifacts required a subset of the available BCD files to be rejected. In the case of asteroid 43032 all IRAC observations failed due to the presence of a close bright background source. For asteroids 11728, 55434, 76019, and 93690, observations in the IRAC short-wavelength channels 1 and 2 failed for the same reason, while observations in channels 3 and 4 succeeded. All remaining observations, including all PUI observations, were successful.

Asteroid fluxes were derived from the final mosaic images using standard synthetic aperture photometry procedures. Different combinations of aperture and sky annulus radii were used together with appropriate aperture correction factors. Statistical flux uncertainties were derived from the sky noise and from the scatter of flux values for different aperture radii (see Mueller, 2007, for more details). In contrast to the thermally-emitted PUI fluxes, the measured IRAC fluxes contain significant amounts of reflected solar radiation. The measured IRAC channel-1 flux $(3.55 \mu \mathrm{m})$ is assumed to be pure reflected sunlight (model calculations showed the thermal component at $3.55 \mu \mathrm{m}$ to be at the few percent level); the reflected solar components in the other IRAC bands were scaled from the channel 1 flux taking the Sun as a black body with $T=$ $5800 \mathrm{~K}$. The mean ratios of thermal flux over total flux for channels 2,3 , and 4 were found to be $0.41 \pm 0.40,0.924 \pm 0.039$, and $0.9950 \pm 0.0014$, respectively. IRAC channel 3 and 4 fluxes were multiplied by these factors to remove the contributions of reflected sunlight. This approach allowed all channel IRAC 3 and 4 observations to be corrected for reflected sunlight, including those for which channel 1 observations failed. IRAC channel 1 and 2 fluxes were not used in the subsequent thermal modeling but we attempted to extract relative reflectance data from them as described below and in Section 5.1. The IRAC channel 1 and 2 measured fluxes are listed in Table 3. 
The flux calibration as provided by the BCD pipelines assumes a nominal source spectrum inversely proportional to wavelength. Since asteroid spectra do not conform to the assumed nominal source spectrum and the filter bandwidths are significant, color corrections have to be applied to the measured thermal fluxes to obtain monochromatic fluxes. Using the measured spectral response curves of the IRAC and PUI systems, we determined color correction factors for a typical MBA thermal emission spectrum on the basis of a thermal model (NEATM with heliocentric distance $=3 \mathrm{AU}$, phase angle $=20^{\circ}$, geometric albedo $p_{\mathrm{v}}=0.2$, "beaming parameter" $\eta=1.0$, see Sect. 3 ). The resulting factors by which the measured thermal fluxes have to be divided are $1.223,1.131$, and 1.089 for IRAC channels 2 - 4 and $0.981,0.971$ for the PUI $15.8 \mu \mathrm{m}$ and $22.3 \mu \mathrm{m}$ channels, respectively. Color-correction factors for the reflected components of the IRAC fluxes are insignificant and have not been applied.

Final corrected flux values for the four thermal-IR channels used for thermal modeling are given in Tables 4 and 5. Tabulated errors reflect only the statistical uncertainties in the flux derivation from the synthetic aperture procedure; absolute calibration uncertainties, systematic instrumental and lightcurve effects may also contribute to the error budget.

While the IRAC channels 1 and 2 flux data were not used in the thermal modeling, we attempted to derive estimates of relative reflectances at the IRAC channel 1 and 2 wavelengths to check for any indications of interesting spectral properties. The thermal components of the fluxes were calculated from the model fits described in the following section, color corrected by multiplying by 1.392 and 1.223 for channels 1 and 2 , respectively, and subtracted from the measured total fluxes to give the measured reflected components. The measured reflected components were ratioed to calculated reflected fluxes based on the adopted $H$-values and the observational geometry (Table 1) assuming the slope parameter $G=0.23$ (see following 
section), the solar $\mathrm{V}$ magnitude $=-26.74$, a solar flux at $3.6 \mu \mathrm{m}$ of $5.546 \mathrm{x} 10^{16} \mathrm{mJy}$ (Gueymard, 2004), a solar black-body temperature of $5800 \mathrm{~K}$ in the wavelength region of channels 1 and 2, and a flat visible-infrared spectral response for the asteroid surfaces. The resulting relative reflectances are listed in Table 3 and discussed in Section 5.1.

\section{Thermal-model fitting}

\subsection{Applicable models}

The thermal emission observed at distance $d$ from an atmosphereless spherical body is given by:

$$
F(\lambda)=\varepsilon R^{2} / d^{2} \iint \mathrm{B}[\lambda, T(\theta, \varphi)] \cos ^{2} \varphi \cos (\theta-\alpha) \mathrm{d} \theta \mathrm{d} \varphi
$$

where $\varepsilon$ is the emissivity, $R$ the radius of the object, B the Planck function, $\varphi$ the latitude, $\theta$ the longitude measured from the sub-solar point, and $\alpha$ the solar phase angle (e.g. Delbo' and Harris, 2002, and references therein). As is usual for asteroids we adopt $\varepsilon=0.9$ throughout this work. Use of Eq. 1 requires a model of the temperature distribution over the surface. The standard thermal model, STM (e.g. Lebofsky et al., 1986, and references therein), is the basic and most widely used asteroid thermal model. The STM may be an appropriate model if the asteroid is rotating slowly and has a low thermal inertia (or its rotation axis points to the Sun) so that each surface element can be considered to be in instantaneous thermal equilibrium with insolation. Objects with significant loose regolith having low heat capacity in poor thermal contact with the bulk mass of the object can be expected to have a low thermal inertia. A corresponding simple model for the extreme case of high thermal inertia and/or fast rotation is 
the so-called fast rotating or isothermal latitude model (Veeder et al., 1989; Lebofsky and Spencer, 1989), hereafter ILM, in which the surface temperature distribution is a function of latitude only. An asteroid with a surface of bare rock would be expected to have a high thermal inertia.

The near-Earth asteroid thermal model, NEATM (Harris, 1998), an extension of the STM, can provide useful estimates of diameter and albedo in cases that are intermediate to those for which the STM and ILM are applicable. While the NEATM was originally devised with nearEarth objects in mind, it is equally applicable to any atmosphereless body. The STM and the NEATM both incorporate a so-called "beaming parameter", $\eta$, which was originally introduced to allow the model temperature distribution to be modified from that of a smooth, zerothermal-inertia sphere to take account of the observed enhancement of thermal emission at small solar phase angles due to surface roughness ("beaming"), i.e:

$$
T(\theta, \varphi)=T_{\mathrm{ss}} \cos ^{1 / 4}(\varphi) \cos ^{1 / 4}(\theta)
$$

The sub-solar temperature, $T_{\mathrm{ss}}=T_{(\theta=0, \varphi=0)}$, is given by

$$
T_{\mathrm{ss}}=[(1-A) S /(\eta \varepsilon \sigma)]^{1 / 4},
$$

where $A$ is the bolometric Bond albedo, $S$ the solar flux at the asteroid, and $\sigma$ the StefanBoltzmann constant. $A$ is related to the geometric albedo, $p_{\mathrm{v}}$, and phase integral, $q$, via $A \approx A_{\mathrm{v}}=$ $q p_{\mathrm{v}}$ (Lebofsky and Spencer, 1989). The phase integral is related to $G$, the slope parameter, via:

$$
q=0.290+0.684 G
$$


(see Wisniewski et al., 1997 and references therein). Following Wisniewski et al. we adopt $G=$ 0.23 throughout this work. The temperature is assumed to fall to zero at the terminator and there is no thermal emission from the night side.

In the refined STM of Lebofsky et al. (1986), designed for use with large main-belt asteroids and requiring measurements at only one thermal-infrared wavelength, $\eta$ has the fixed value of 0.756. In contrast, the NEATM treats $\eta$ as a variable, which modifies the color temperature, or flux distribution, of the model thermal continuum and enables it to be accurately fitted to the measured continuum flux distribution. The price paid for the higher accuracy of the NEATM is the requirement for thermal-infrared measurements at more than one wavelength. A variable $\eta$ allows a first-order correction for effects such as beaming, thermal inertia and rotation that influence the surface temperature distribution presented to the observer. In the case of the NEATM the value of $\eta$ giving the best fit to the measured continuum flux distribution is found via an iterative procedure.

The best-fit value of $\eta$ resulting from application of the NEATM is a measure of the extent to which the apparent surface temperature distribution departs from that of a perfect STM-type asteroid (i.e. smooth, with zero thermal inertia), for which $\eta=1$. At small or moderate phase angles, such as those at which our targets are observed, $\eta>1$ indicates significant thermal inertia, whereas $\eta<1$ indicates significant beaming due to surface roughness. The NEATM takes account of the phase angle by numerically integrating the observable thermal emission from the spherical surface illuminated by the Sun, assuming that surface emits with a Lambertian angular distribution.

For more detailed discussions of the NEATM and other thermal models outlined here see Harris (1998), Delbo' and Harris (2002), Harris and Lagerros (2002), Delbo'et al. (2003), and 
Harris (2006), and references therein. Delbo'and Harris (2002) give the mathematical expressions for calculating the wavelength-dependent observable thermal-infrared fluxes for all three models.

\subsection{Rotational variability}

It is important to take account of the rotational variability in observational data when applying thermal models. With simple models based on spherical geometry, optical lightcurves are often used as the basis for correcting thermal-infrared data, a practice that can introduce errors if the optical and thermal-infrared lightcurves are out of phase or differ in structure (e.g. Harris et al., 2005). Differences in the lightcurves of reflected and emitted radiation can arise from the effects of shape, surface structure and thermal inertia. Rotational variability of the flux values can alter the relative measurements obtained at different wavelengths and therefore influence the results from the model fitting, e.g. lead to erroneous values of $\eta$ in the case of the NEATM. In the present case the short time delays (typically $20 \mathrm{~min}$ ) between IRAC observations in bands and $1 \& 3$ and $2 \& 4$, and between the two PUI bands (typically $1-2 \mathrm{~min}$ ), compared to the rotation periods of our targets (5-33 h; see Table 1$)$, lead to little non-statistical variability between measurements in the coupled filters, and only minor errors in color temperature derivation. However, the time difference between observations with IRAC and observations with IRS PUI was often of the order of weeks, leaving open the possibility that $\eta$ values derived via model fitting to all four thermal flux values simultaneously could be subject to large errors due to rotational lightcurve effects. Therefore, to investigate the magnitude of systematic errors introduced by rotation into the albedo and diameter results, we examined the results of model fitting to the IRAC and IRS PUI fluxes taken separately, before performing four-measurement fits, as discussed in the following section. 


\section{Results and Analysis}

Since the IRAC and IRS PUI measurements were separated in time by days or weeks, i.e. at least a few rotational periods, we first carried out a check for rotational lightcurve effects by fitting NEATM to the fluxes from these instruments independently, and then comparing the derived IRAC and PUI diameters. The rotation of the targets could significantly affect the cross-sectional area presented to the observer and thus the derived size of an object, but would not be expected to influence $\eta$ significantly (unless there were large differences in thermal inertia or surface roughness on a hemispherical scale, which we assume to be unlikely on such small objects). Therefore at this stage we used the mean of $\eta_{\text {IRAC }}$ and $\eta_{\text {PUI }}$ as the best estimate of $\eta$. In most cases the diameter discrepancies are less than, or do not greatly exceed, $10 \%$; exceptions being amongst the smallest targets with the largest fractional flux uncertainties, namely 55434, 75176, 76019, and 93690 (Table 7). The fact that there is little apparent correlation with the available lightcurve amplitude data (Table 1) suggests that in general the additional uncertainty due to lightcurve effects is relatively small.

We take the reasonable overall agreement between the results from IRAC and IRS PUI (Table 6) as justification of our final analysis, which consisted of fitting NEATM simultaneously to four flux measurements (IRAC $5.731 \mu \mathrm{m}, 7.872 \mu \mathrm{m}$; IRS PUI $15.8 \mu \mathrm{m}, 22.3 \mu \mathrm{m}$ ) after normalizing the PUI flux measurements to the observing geometry of the IRAC measurements using the NEATM, i.e. adjusting the PUI fluxes for the slightly different heliocentric and Spitzer-centric distances and solar phase angles. Fitting to four flux measurements together minimizes the influence of residual uncertainties in individual flux measurements, so the results of this approach should be significantly more accurate than those of the two- 
measurement fits given in Table 6; indeed the general high quality of the fits (Fig. 1) supports this conclusion.

Uncertainties in thermal modeling usually exceed the formal errors resulting from the scatter of the flux measurements. Wright (2007) has tested the NEATM against a sophisticated thermophysical model and finds that it gives diameter estimates that are accurate to $10 \% \mathrm{RMS}$ for phase angles less than $60^{\circ}$, even for the non-spherical shapes typical of, for example, nearEarth objects. Similar results were obtained by Delbo'(2004). In the case of a very irregularly shaped object, or one with complex large-scale surface topography, errors may be larger. Given the good quality of the Spitzer data set discussed in this work, and the relatively low solar phase angles of the target asteroids, an overall uncertainty of $10 \%$ in the derived diameters is a conservative estimate in most cases.

The uncertainty in the adopted $H$ values increases the uncertainty in the derived values of albedo but has little effect on the uncertainty in the diameter (Harris and Harris, 1997). For the albedo results we assume a $20 \%(1 \sigma)$ uncertainty (albedo scales as the inverse square of diameter for constant $H$ ) plus an additional component added in quadrature due to a typical 0.3 mag uncertainty in the $H$ values, giving an overall uncertainty estimate of $37 \%(1 \sigma)$. Should improved $H$ values become available in the future, our albedo results can be easily updated using the method of Harris and Harris (1997).

The error estimates for $\eta$ were derived by means of a Monte Carlo routine, which fits the NEATM to flux values generated randomly within a Gaussian distribution consistent with the $1 \sigma$ uncertainties in the measured values. 
The final diameter, albedo and $\eta$ values are given in Table 7. Representative plots of the final corrected fluxes and NEATM fits are shown in Fig. 1.

\section{Discussion}

\subsection{Relative reflectances}

The relative reflectances given in Table 3 result from dividing the measured reflected flux components by fluxes calculated from the V-band brightnesses and the solar flux distribution assuming a flat spectral response of the asteroid surfaces, as described in Section 2. The uncertainties in the tabulated relative reflectances are considerable: In the case of IRAC channel 1 they are dominated by the uncertainty in the optical brightness of the asteroid ( $H$ value), which is around $30 \%$ for a 0.3 mag uncertainty in $H$; the uncertainties in the IRAC 1 measured fluxes are normally much less than this (Table 3). In the case of IRAC channel 2 we add a further $30 \%$ in quadrature for the estimated uncertainty in the difference of the total measured flux and the thermal component. The resulting estimated uncertainties are around $40 \%$ for the IRAC 2 band and somewhat less for IRAC 1 . Given the uncertainties, it is arguable whether the variations from object to object are significant. However, the means and standard deviations of the derived relative reflectances are $1.39 \pm 0.23$ (channel 1) and $1.11 \pm$ 0.35 (channel 2), which provide significant evidence for spectral reddening between the $\mathrm{V}$ band and the channel 1 wavelength of $3.55 \mu \mathrm{m}$. While the channel 2 relative reflectances are highly uncertain, there appears to be a slight blue slope in general between channels 1 and 2 (3.55 $\mu \mathrm{m}$ and $4.5 \mu \mathrm{m}$ ): the mean and standard deviation of the channel $2 / 1$ relative reflectance ratio are $0.81 \pm 0.25$ (note that this ratio is virtually unaffected by the uncertainty in $H$ ). We 
find no evidence for correlations of spectral reflectance, or spectral slope, with size, albedo, or $\eta$.

While the available spectral data for asteroids in this wavelength region are sparse, we note that Rivkin et al. (1997) report that the $2.95-\mu \mathrm{m} / 0.55-\mu \mathrm{m}$ reflectance ratios of 7 main-belt Sclass asteroids cluster very close to 1.4 , which is consistent with our mean relative reflectance at $3.55 \mu \mathrm{m}$. Furthermore, model calculations for large grains of olivine and pyroxene (e.g. Emery and Brown, 2004) suggest $S$ types should have a roughly neutral spectrum in the $3.5 \mu \mathrm{m}$ - $4.5 \mu \mathrm{m}$ range, consistent with our result given the uncertainties. We conclude that the measured reflectivities are consistent with expectations for S-type asteroids. A more detailed comparison of our spectral reflectance results with possible spectral features is beyond the scope of the present paper; we include the data here for completion and in anticipation of their possible usefulness for future studies.

\subsection{Albedos}

The resulting albedo distribution of the target Karin asteroids is shown in Fig. 2. In all cases the albedo is within the expected range for S-type asteroids and the mean albedo, excluding 832 and 43032 is $p_{\mathrm{v}}=0.215 \pm 0.015$, with a standard deviation of 0.057 , which is very similar to the albedo of 832 Karin $\left(p_{\mathrm{v}}=0.20 \pm 0.07\right)$. By definition the Karin cluster asteroids are dynamically related; these albedo results are strongly suggestive of a physical relationship as well between the Karin cluster asteroids and 832 Karin.

The albedo distribution (Fig. 2) appears to have an extended tail at the high albedo end, suggesting that a number of Karin cluster members have albedos that are significantly higher than that of 832 Karin. While the significance of this result is questionable given the small 
number of asteroids in our data set and the uncertainties in the derived albedos, it may reflect differences in the degree of space weathering due to exposure to the solar wind and micrometeorite bombardment (space weathering appears to darken the surfaces of olivine-rich taxonomic types, such as S and Q types; Clark et al., 2002). Further insight into this possibility is gained by comparing the albedo versus diameter distribution of the Karins (Fig. 3a) with that of S- and Q-type near-Earth asteroids (Fig. 3b). In the case of the near-Earth asteroids (NEAs) there appears to be a trend of increasing albedo with decreasing diameter (Harris, 2006; Delbo' et al., 2003), which is consistent with the idea that collisional processing leads to reduced lifetimes for small NEAs and therefore reduced exposure to space weathering. Since the Karin cluster asteroids presumably all have the same age and have suffered relatively few collisions since formation, a similar dependence of albedo on diameter would not be expected, consistent with the results plotted in Fig. 3a. However, it is interesting to note that the albedos of the Karins are at the lower end of the albedo range of the NEAs, indicating that despite their relatively low age $(\sim 5.8 \mathrm{Myr})$ they may have undergone significant darkening due to space weathering. Our results support the conclusions of others (e.g. Chapman et al., 2007) that space-weathering effects on 832 Karin are already in evidence, despite its age of only $\sim 5.8$ Myr.

The mean albedo of 0.215 is very similar to the nominal value of 0.2 assumed by Nesvorný et al. (2006), based on albedos of Koronis family members given in the IRAS SIMPS catalog (Tedesco et al., 2002). Our Spitzer results appear to provide evidence that the Karin cluster members have similar albedos to the Koronis family and confirm the legitimacy of the albedo and size assumptions made in the Karin cluster formation modeling of Nesvorný et al. (2006).

While at first sight it is reassuring that the Karin cluster and Koronis family have similar albedos, since the Karin cluster is thought to have originated in the break up of a Koronis 
family member, the question arises as to why the much older ( 2 Gyr; e.g. Marzari et al., 1995) Koronis member surfaces are not darker than the presumably less space-weathered Karin-cluster members. On the one hand we note that the IRAS fluxes for many of the Koronis family asteroids are very uncertain and it is questionable to what extent IRAS albedos in the size range of the Koronis asteroids listed in the SIMPS catalog $(15-40 \mathrm{~km})$ are trustworthy (Tedesco et al., 2002, quote the uncertainty of IRAS diameters of objects in the size range 100$350 \mathrm{~km}$ as $10 \%$; the corresponding uncertainty in the albedos of these much larger objects is about $20 \%$ ). On the other hand it appears possible that space weathering may reach equilibrium with impact gardening over a timescale as short as several million years. Vernazza et al. (2006) report evidence of space weathering in the spectra of the majority of their Karin cluster targets. Chapman et al. (2008) find only slight differences between infrared reflectance spectra of Karin and Koronis asteroids and note that the deep absorption bands expected for nonweathered mineral assemblages are absent in both cases. If the Koronis albedos can be taken at face value, our results support the view that space weathering in the main belt can proceed to completion on a rather short timescale of less than 10 million years, at least as far as surface darkening is concerned.

\section{3. $\eta$ values: surface roughness}

Given the error bars, the spread of $\eta$ values (Figs. 4, 5, and 6) appears to be very significant. The value of $\eta$ that results from NEATM fitting to the thermal-infrared flux measurements of an asteroid is a measure of the extent to which the thermal properties of the asteroid depart from those of an ideal spherical, smooth object with zero thermal inertia (or a sunward-oriented rotation axis), i.e. the properties assumed in the STM. There appears to be a sharp cut-off at the low end of the $\eta$ range, consistent with the value of 0.756 derived by Lebofsky et al. (1986) in their refined STM. As discussed in Section 3, values of $\eta$ significantly below unity indicate the 
presence of "beaming", or enhanced thermal emission in the sunward direction, normally assumed to be due to surface roughness resulting from cratering. On the other hand, at low or moderate phase angles, such as those of our target asteroids (Table 1), values of $\eta$ significantly above unity require surface temperatures on the sunward side below those that would result from instantaneous thermal equilibrium, indicating significant thermal inertia, i.e. a less thermally-insulating regolith. It appears, therefore, that our target asteroids differ significantly in terms of their surface roughness and regolith properties. While Figs. 4 and 5 reveal no striking dependence of size or albedo on $\eta$, it is interesting to note that the four objects in Fig. 5 with $\eta$ values significantly above unity all have albedos at the lower end of the albedo range (see also Table 7). On the other hand the lowest values of $\eta$ are associated predominantly with relatively high albedos. While over-interpretation of the albedo data is a danger in light of the uncertainties, Fig. 5 may provide further evidence of rapid space weathering of the Karin cluster asteroids: objects which happened statistically to have suffered more impacts since their formation 5.8 Myr ago would have rougher surfaces, resulting in lower $\eta$ values, and also partially lighter surfaces due to the exposure of sub-surface, non-space-weathered material via impact gardening. Objects which have suffered fewer impacts would have smoother surfaces, resulting in higher $\eta$ values, and retain more space-weathered, darker material on their surfaces. This scenario requires similar timescales of some $10 \mathrm{Myr}$ for space-weathering of the Karin cluster and several regolith-churning impacts on 1 - $5 \mathrm{~km}$-sized asteroids.

\section{4. $\eta$ values: thermal inertia}

It is also possible to make some inferences about thermal inertia and regolith properties from the derived $\eta$ values. As mentioned above, $\eta$ values above unity are indicative of significant thermal inertia. Given knowledge of an object's rotation rate but no information on spin-axis orientation, it is possible to derive a rough lower limit for the thermal inertia. Using a smooth- 
sphere thermophysical model we derived the thermal inertia implied by the $\eta$ values of 40921 , for which a rotation period is available, and 76019, which has the largest $\eta$ value of all our target asteroids. In the case of $40921(\eta=1.11)$ the measured spin period is $6.74 \mathrm{hr}$ (Table 1$)$. In the case of $76019(\eta=1.19)$ we assumed a period of $20 \mathrm{hr}$, which is comparable with the longest periods in Table 1; shorter periods result in smaller values of thermal inertia (with constant $\eta$ the derived thermal inertia varies as the square root of the period). The calculation assumes the spin axis is oriented perpendicular to the solar direction; if this is not the case the implied thermal inertia can be much larger than that calculated. Furthermore, the beaming effect reduces $\eta$, so the presence of surface roughness also leads to underestimation of thermal inertia.

For 40921 we obtain thermal inertia $\Gamma>15 \mathrm{Jm}^{-2} \mathrm{~s}^{-0.5} \mathrm{~K}^{-1}$, while for 76019 with an assumed rotation period of $20 \mathrm{~h}$ we obtain thermal inertia $\Gamma>48 \mathrm{Jm}^{-2} \mathrm{~s}^{-0.5} \mathrm{~K}^{-1}$. These values are consistent with those for regolith-covered large main-belt asteroids, whereas typical values for small NEAs in the size range of our Karin cluster targets are much higher, of the order 150 $300 \mathrm{Jm}^{-2} \mathrm{~s}^{-0.5} \mathrm{~K}^{-1}$ (e.g., Delbo'et al., 2007; Harris, 2006; Harris, et al., 2005; and references therein). Given our lack of knowledge of the spin vectors, shapes and surface characteristics of the Karin asteroids, it is quite possible that the thermal inertias of 40921 and 76019 are actually much larger. However, it appears to be significant that amongst our set of 16 targets for which reliable $\eta$ values have been determined, we find no asteroids with $\eta$ indicative of the higher thermal inertia values that have been reported for NEAs with similar sizes (e.g., if 76019 had thermal inertia $=200 \mathrm{Jm}^{-2} \mathrm{~s}^{-0.5} \mathrm{~K}^{-1}$, an $\eta$ value of up to 1.75 would be expected; cf. the $\eta$ values in Table 7).

With a sample of 16 objects we would expect a few to have their spin axes orientated by chance nearly perpendicular to the solar direction. Even under the assumption of a very long 
rotation period of $20 \mathrm{~h}$, the histogram in Fig. 6 shows that the distribution of $\eta$ values falls far short of that expected for a sample of asteroids with random spin-axis orientations and thermal inertia values up to $\Gamma=200 \mathrm{Jm}^{-2} \mathrm{~s}^{-0.5} \mathrm{~K}^{-1}$. Some reduction in the $\eta$ values may result from surface roughness and the beaming effect, which explains why many $\eta$ values in Table 7 are less than 1.0, the theoretical minimum value for $\Gamma=0$ in the smooth-sphere standard thermal model. However, the lowest value of $\eta$ in Table 7 is 0.76 , near the minimum for realistic very rough surfaces (Spencer, 1990), suggesting a maximum reduction in $\eta$ of only 0.24 .

Since NEAs are considered to be fragments from the main belt, we do not expect any fundamental physical differences between them and similarly-sized main-belt asteroids. Why then do we not see evidence of higher thermal inertia amongst the Karins? One possibility is that our target set is simply too small and contains a non-representative sample. Alternatively, the reason may lie with effects that can cause NEAs to lose regolith. We speculate that NEAs with their smaller heliocentric distances $(\sim 1 \mathrm{AU})$ are more strongly affected by the YORP effect and can spin up to rotation rates at which material can be lost from their surfaces on timescales of 1 My or so (Bottke et al., 2006), i.e. much shorter than their dynamical lifetimes. Holsapple (2007, figure 1) shows that there are a number of asteroids with diameters up to 20 $\mathrm{km}$ having periods in the theoretical range of upper limits for materials without cohesive or tensile strength, i.e. 2-3 h. In contrast to the situation for NEAs, the corresponding timescale for spin-up of the Karin asteroids in the main belt at $\sim 3 \mathrm{AU}$ may be longer than their $\sim 6 \mathrm{Myr}$ lifetime (Rubincam, 2000) so they could have retained regolith until the present time.

We note that the results of Nesvorný and Bottke (2004), who examined the Yarkovsky effect on the Karins, are consistent with the presence of thermally-insulating regolith. 
Future determinations of spin periods and spin-axis orientations of the Karin cluster members will enable more robust conclusions on thermal inertia to be drawn on the basis of the Spitzer data presented here.

\section{Conclusions}

On the basis of thermal-infrared observations with the Spitzer Space Telescope we have made the first direct determinations of sizes and albedos for 17 main-belt asteroids belonging to the Karin cluster, including the largest member, 832 Karin. In all cases derived albedos are within the expected range for S-type asteroids and the mean albedo, excluding 832 and 43032 is $p_{\mathrm{v}}=$ $0.215 \pm 0.015$, with a standard deviation of 0.057 , which is very similar to the albedo we find for 832 Karin $\left(p_{\mathrm{v}}=0.20 \pm 0.07\right)$. Our albedo results provide further evidence in favor of a physical relationship between the cluster members and 832 Karin, and constitute the first observational confirmation of the legitimacy of assumptions made by Nesvorný et al. (2006) in recent modeling of the formation of the Karin cluster via a single catastrophic collision $5.8 \pm$ 0.2 Myr ago.

The Karin cluster albedos show no dependence with size over the limited range of size covered, and are at the lower end of the albedo range of near-Earth asteroids of similar taxonomic types, suggesting that the Karin cluster asteroids have undergone significant space weathering since their formation only 5.8 Myr ago. Furthermore, space weathering may be the reason for the association of low $\eta$ values with relatively high albedos and vice versa, suggesting a link between roughness and lighter surfaces: A statistically greater number of impacts on some objects may have resulted in higher albedos due to impact gardening. 
No evidence has been found for thermal inertia values significantly higher than those previously reported for large main-belt asteroids, despite the order-of-magnitude higher values reported for some NEAs with similar sizes to the asteroids in this study. These results are consistent with the conclusion of Nesvorný and Bottke (2004) that the Karin cluster members are covered by a regolith layer of low thermal conductivity. We speculate that the YORP effect can spin up NEAs to near rotational disruption on shorter timescales than main-belt asteroids due to the lower heliocentric distances of NEAs, causing them to lose regolith. We caution, however, that we are able to derive lower limits to thermal inertia only; accurate thermal inertia determinations for the Karin cluster asteroids require more precise information on their spin vectors and shapes.

\section{Acknowledgments}

This work is based on observations made with the Spitzer Space Telescope, which is operated by the Jet Propulsion Laboratory, California Institute of Technology under a contract with NASA. Liberal use was made of the excellent JPL Horizons System for generating ephemerides. We wish to thank Dave Osip for the provision of lightcurve amplitude estimates, Josh Emery and Andy Rivkin for useful discussions, and the referees, Clark Chapman and anonymous, for their incisive comments. 


\section{References}

Binzel, R.P., 1987. A photoelectric survey of 130 asteroids. Icarus 72, 135-208.

Bottke, W.F., Vokrouhlicky, D., Rubincam, D.P., Nesvorný, D., 2006. The Yarkovsky and YORP effects: implications for asteroid dynamics. Ann. Rev. Earth Planet. Sci. 34, 157-191

Chapman, C.R., Enke, B., Merline W.J., Tamblyn, P., Nesvorný, D., Young, E.F., Olkin, C., 2007. Young asteroid 832 Karin shows no rotational spectral variations. Icarus 191, $323-329$.

Chapman, C.R., Enke, B., Merline W.J., Nesvorný, D.,Tamblyn, P., Young, E.F. 2008. Reflectance spectra of very young asteroid families. Asteroids, Comets, Meteors ACM 2008. Abstract 8391 .

Cheng, A., 2004. Collisional evolution of the asteroid belt. Icarus 169, 357-372.

Clark, B.E., Hapke, B., Pieters, C., Britt, D., 2002. Asteroid space weathering and regolith evolution. In: Bottke, W.F., Cellino, A., Paolicchi, P., and Binzel, R.P. (Eds.), Asteroids III. Univ. of Arizona Press, Tucson, pp. 585-599.

Davis, D.R., Durda, D.D., Marzari, F., Campo Bagatin, A., and Gil-Hutton, R., 2002. Collisional evolution of small-body populations. In: Bottke, W.F., Cellino, A., 
Paolicchi, P., Binzel, R.P. (Eds.), Asteroids III. Univ. of Arizona Press, Tucson, pp. 545 -558 .

Delbo', M., 2004. The nature of near-Earth asteroids from a study of their thermal-infrared emission. Doctoral dissertation, Free University of Berlin. http://www.diss.fuberlin.de/2004/289/indexe.html

Delbo', M., Harris, A.W., 2002. Physical properties of near-Earth asteroids from thermal infrared observations and thermal modeling. Meteoritics and Plan. Sci. 37, 1929-1936.

Delbo', M., Harris, A.W., Binzel, R.P, Pravec, P., Davies, J.K., 2003. Keck observations of near-Earth asteroids in the thermal infrared. Icarus 166, 116-130.

Delbo', M., dell'Oro, A., Harris, A.W., Mottola, S., Mueller, M., 2007. Thermal inertia of near-Earth asteroids and implications for the magnitude of the Yarkovsky effect. Icarus 190, 236-249.

Emery, J.P., Brown, R.H., 2004. The surface composition of Trojan asteroids: constraints set by scattering theory. Icarus, 170, 131-152.

Fazio, G.G., Hora, J.L., Allen, L.E., and 62 colleagues, 2004. The Infrared Array Camera (IRAC) for the Spitzer Space Telescope. Astrophys. J. Suppl. 154, 10-17.

Gueymard, C.A., 2004. The sun's total and spectral irradiance for solar energy applications and solar radiation models. Solar Energy 76, 423-453. 
Hahn, G., Mottola, S., Sen, A. K., Harris, A.W., Kührt, E., Müller, M. 2006. Photometry of Karin family asteroids. Bull. Astron. Soc. of India 34, 393-399.

Harris, A.W., 1998. A thermal model for near-Earth asteroids. Icarus 131, 291-301.

Harris, A.W., 2006. The surface properties of small asteroids from thermal-infrared observations. In: Lazzaro, D., Ferraz-Mello, S., Fernández, J.A. (Eds.), Proc. of IAU Symposium 229. Cambridge University Press, Cambridge, pp. 449-463.

Harris, A.W., Harris, A.W., 1997. On the revision of radiometric albedos and diameters of asteroids. Icarus 126, 450-454.

Harris, A.W., Lagerros, J.S.V., 2002. Asteroids in the thermal infrared. In: Bottke, W.F., Cellino, A., Paolicchi, P., Binzel, R.P. (Eds.), Asteroids III. Univ. of Arizona Press, Tucson, pp. 205-218.

Harris, A.W., Mueller, M., Delbo', M., Bus, S. J., 2005. The surface properties of small asteroids: peculiar Betulia - a case study. Icarus 179, 95-108.

Holsapple, K.A., 2007, Spin limits of Solar System bodies: From the small fast-rotators to 2003 EL61. Icarus 187, 500-509.

Houck, J.R, Roellig, T.L., van Cleve, J., Forrest, W.J., Herter, T., Lawrence, C.R., Matthews, K., Reitsema, H.J., Soifer, B.T., Watson, D.M., Weedman, D., Huisjen, M., Troeltzsch, J., Barry, D.J., Bernard-Salas, J., Blacken, C.E., Brandl, B.R., Charmandaris, V., Devost, D., Gull, G.E., Hall, P., Henderson, C.P., Higdon, S.J.U., Pirger, B.E., 
Schoenwald, J., Grillmair, C.J., Ingalls, J.G., Morris, P.W., Teplitz, H.I., 2004. The Infrared Spectrograph (IRS) on the Spitzer Space Telescope. Astrophys. J. Suppl. 154, $18-24$.

Lebofsky, L.A., Spencer, J.R., 1989. Radiometry and thermal modeling of asteroids. In: Binzel, R.P., Gehrels, T., Matthews, M. S., (Eds.), Asteroids II. Univ. of Arizona Press, Tucson, pp. 128-147.

Lebofsky, L.A., Sykes, M.V., Tedesco, E.F., Veeder, G.J., Matson, D.L., Brown, R.H., Gradie, J.C., Feierberg, M.A., Rudy, R.J., 1986. A refined "standard" thermal model for asteroids based on observations of 1 Ceres and 2 Pallas. Icarus 68, 239-251.

Lisse, C., Beichman, C.A., Bryden, G., Wyatt, M.C., 2007. On the nature of the dust in the debris disk around HD 69830. Astrophys. J. 658, 584-592.

Makovoz, D., Khan, I., 2005. Mosaicking with MOPEX. In: Shopbell, P.L., Britton, M.C., Ebert, R., (Eds.), Astronomical Data Analysis Software and Systems XIV ASP Conf. Ser., vol. 347. Astron. Soc. of the Pacific, San Francisco, pp. 81-85.

Mueller, M., 2007. Surface properties of asteroids from mid-infrared observations and thermophysical modeling. Doctoral dissertation, Free University of Berlin. http://www.diss.fu-berlin.de/2007/471/indexe.html

Nesvorný, D., Bottke, W. F., 2004. Detection of the Yarkovsky effect for main-belt asteroids. Icarus $170,324-342$. 
Nesvorný, D., Bottke, W. F., Jr., Dones, L., Levison, H. F., 2002. The recent breakup of an asteroid in the main-belt region. Nature 417, 720-771.

Nesvorný, D., Enke, B.L., Bottke, W. F., Durda, D.D., Asphaug, E., Richardson, D.C., 2006. Karin cluster formation by asteroid impact. Icarus 183, 296-311.

Rivkin, A.S., Howell, E.S., Clark, B.E., Lebofsky, L.A., Britt, D.T., 1997. S-class asteroids: the view from three microns. Lunar and Planet. Sci. 28. Abstract 1803.

Rubincam, D.P., 2000. Radiative spin-up and spin-down of small asteroids. Icarus 148, 2-11.

Spencer, J.R., 1990. A rough-surface thermophysical model for airless planets. Icarus 83, $27-$ 38.

Tedesco, E.F., Noah, P.V., Noah, M., Price, S.D., 2002. The supplemental IRAS minor planet survey. Astron. J. 123, 1056-1085.

Veeder, G. J., Hanner, M.S., Matson, D.L., Tedesco, E.F., Lebofsky, L.A., Tokunaga, A.T., 1989. Radiometry of near-Earth asteroids. Astron. J. 97, 1211-1219.

Vernazza, P., Birlan, M., Rossi, A., Dotto, E., Nesvorný, D., Brunetto, R., Fornasier, S., Fulchignoni, M., Renner, S., 2006. Physical characterization of the Karin family. Astron. and Astrophys. 460, 945-951.

Werner, M., Roellig, T., Low, F., Rieke, G., Rieke, M., Hoffmann, W., Young, E., Houck, J., Brandl, B., Fazio, G., Hora, J., Gehrz, R., Helou, G., Soifer, B., Stauffer, J., Keene, J., 
Eisenhardt, P., Gallagher, D., Gautier, T., Irace, W., Lawrence, C., Simmons, L., Van

Cleve, J., Jura, M., Wright, E., 2004. The Spitzer Space Telescope Mission. Astrophys. J. Suppl. 154, 1-9.

Wisniewski, W.Z., Michałowski, T.M., Harris, A.W., McMillan, R.S., 1997. Photometric observations of 125 asteroids. Icarus 126, 395-449.

Wolters, S.D., Green, S.F., McBride, N., Davies, J.K., 2008. Thermal infrared and optical observations of four near-Earth asteroids. Icarus 193, 535-552.

Wright, E.L., 2007. Comparing the NEATM to a rotating, cratered thermophysical asteroid model. Bull. Am. Astron. Soc. 39. Abstract \#35.11. 


\section{TABLE 1}

Observing parameters, observing geometry, and lightcurve parameters

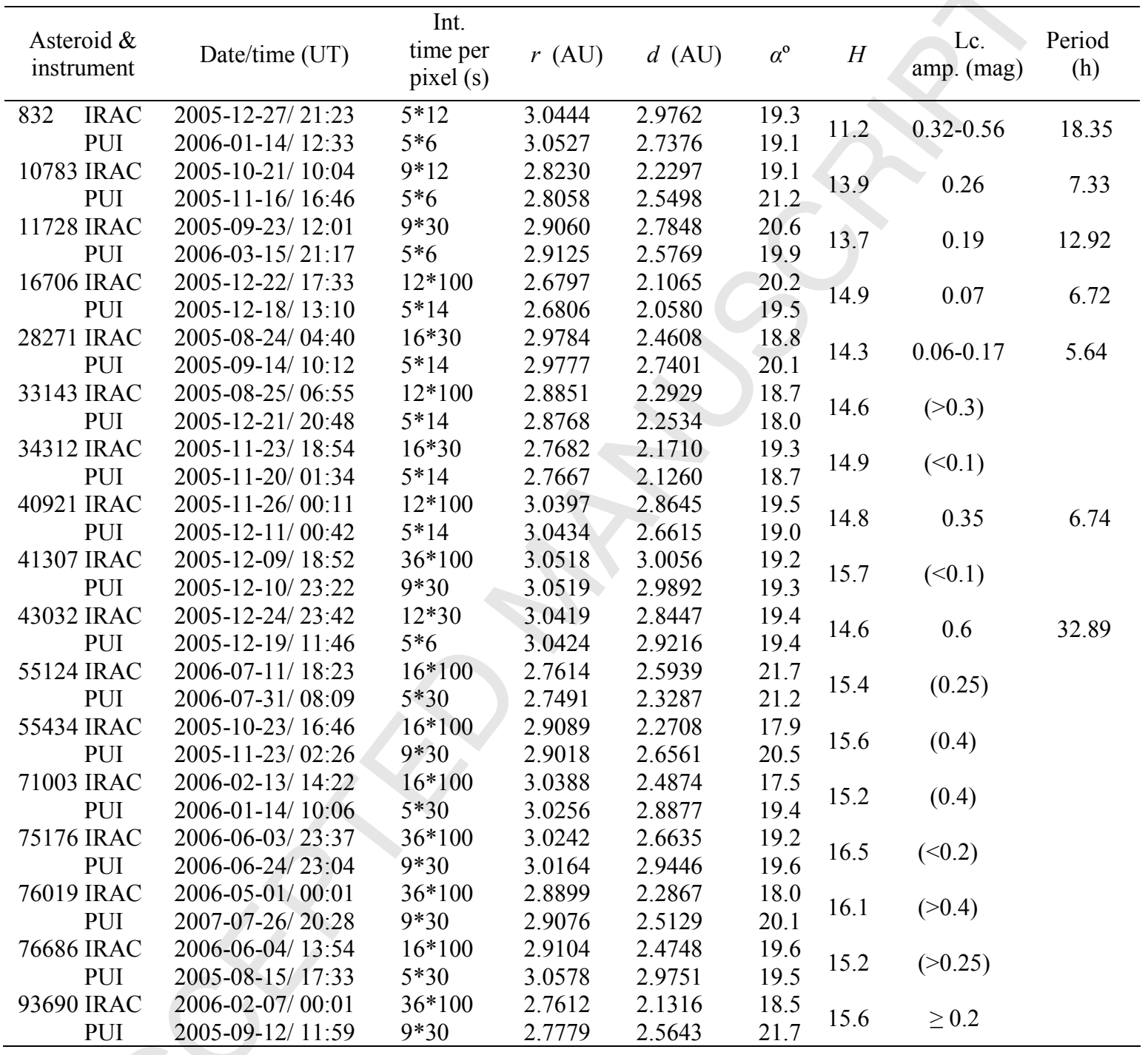

Note: $r=$ heliocentric distance; $d=$ distance from Spitzer; Lc. amp. = lightcurve amplitude. IRAC channels $1+3$ and channels $2+4$ exposures are separated in time by typically $20 \mathrm{~min}$; the times given are the approximate mean start times of the IRAC exposures in each case. The IRS PUI 16 and $22 \mu \mathrm{m}$ exposures were executed back-toback, and are between 30s and 5 min each; the times given are the approximate mean start times of the 16 and 22 $\mu \mathrm{m}$ exposures in each case. Integration times are given as number of on-target frames multiplied by the integration time per frame. See Table 2 for adopted $H$ values. Lightcurve parameters for 11728 and 93690 are from Hahn et al. (2006), those for 832, 10783, 16706, 28271, 40921, 43032 are from Harris, A.W., Warner, B.D., and Pravec, P., Eds., Asteroid Lightcurve Derived Data. EAR-A-5-DDR-DERIVED-LIGHTCURVE-V9.0. NASA Planetary Data System, 2007. Lightcurve amplitudes in parentheses are estimates from D. Osip (personal communication). 


\section{TABLE 2}

$H$ values for the observed Karins

\begin{tabular}{cccc}
\hline Asteroid & $\begin{array}{c}H \\
\text { Tholen }\end{array}$ & $\begin{array}{c}H \\
\text { other }\end{array}$ & $\begin{array}{c}H \\
\text { adopted }\end{array}$ \\
\hline 832 & 11.18 & 11.26 & 11.2 \\
10783 & 13.9 & & 13.9 \\
11728 & 14.2 & 13.70 & 13.7 \\
16706 & 14.9 & & 14.9 \\
28271 & 14.3 & & 14.3 \\
33143 & 14.6 & & 14.6 \\
34312 & 14.9 & & 14.9 \\
40921 & 14.8 & & 14.8 \\
41307 & 15.7 & & 15.7 \\
43032 & 14.6 & & 14.6 \\
55124 & 15.4 & & 15.4 \\
55434 & 15.6 & & 15.6 \\
71003 & 15.2 & & 15.2 \\
75176 & 16.5 & & 16.5 \\
76019 & 16.1 & & 16.1 \\
76686 & 15.2 & & 15.2 \\
93690 & 15.8 & 15.58 & 15.6 \\
\hline
\end{tabular}

Note: " $H$ other" for 832 from Binzel (1987), For 11728 and 93690 from Hahn et al. (2006). Tholen $H$ values are from Tholen, D.J., Ed., Asteroid Absolute Magnitudes. EAR-A-5DDR-ASTERMAG-V11.0. NASA Planetary Data System, 2007. 


\section{TABLE 3}

IRAC channel 1 and 2 fluxes and derived relative reflectances

\begin{tabular}{|c|c|c|c|c|c|c|}
\hline \multirow{2}{*}{ Asteroid } & \multicolumn{4}{|c|}{ Measured fluxes (mJy) } & \multicolumn{2}{|c|}{$\begin{array}{l}\text { Relative reflectances } \\
\text { (relative to the } \mathrm{V} \text { band) }\end{array}$} \\
\hline & $\begin{array}{l}\text { IRAC } 1 \\
3.55 \mu \mathrm{m}\end{array}$ & Err. 1 & $\begin{array}{l}\text { IRAC } 2 \\
4.493 \mu \mathrm{m}\end{array}$ & Err. 2 & $3.55 \mu \mathrm{m}$ & $4.493 \mu \mathrm{m}$ \\
\hline 832 & 0.2984 & 0.0039 & 0.3277 & 0.0060 & 1.42 & 1.26 \\
\hline 10783 & 0.0622 & 0.0033 & 0.1228 & 0.0045 & 1.56 & 1.00 \\
\hline 11728 & - & - & - & - & & \\
\hline 16706 & 0.0211 & 0.0012 & 0.0366 & 0.0017 & 1.16 & 1.30 \\
\hline 28271 & 0.0254 & 0.0059 & 0.0328 & 0.0052 & 1.34 & 1.25 \\
\hline 33143 & 0.0196 & 0.0027 & 0.0187 & 0.0024 & 1.13 & 0.87 \\
\hline 34312 & 0.0264 & 0.0030 & 0.0423 & 0.0038 & 1.57 & 0.86 \\
\hline 40921 & 0.0121 & 0.0015 & 0.0089 & 0.0009 & 1.49 & 0.92 \\
\hline 41307 & 0.0040 & 0.0008 & 0.0065 & 0.0007 & 1.19 & 1.04 \\
\hline 43032 & - & - & - & - & 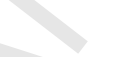 & \\
\hline 55124 & 0.0089 & 0.0010 & 0.0188 & 0.0015 & 1.20 & 0.48 \\
\hline 55434 & - & - & - & & & \\
\hline 71003 & 0.0121 & 0.0026 & 0.0136 & 0.0033 & 1.50 & 1.01 \\
\hline 75176 & 0.0038 & 0.0006 & 0.0044 & 0.0016 & 1.88 & 1.88 \\
\hline 76019 & - & - & & - & & \\
\hline 76686 & 0.0096 & 0.0018 & 0.0125 & 0.0033 & 1.16 & 1.45 \\
\hline 93690 & - & - & & - & & \\
\hline
\end{tabular}

Note: The errors reflect only the statistical uncertainties in the flux derivation from the synthetic aperture procedure; absolute calibration uncertainties, systematic instrumental and lightcurve effects may also contribute to the error budget. To obtain relative reflectance the thermal emission component in each case, calculated from the model fitting described in Section 3, was subtracted from the measured flux and the result divided by the expected reflected component based on the $H$ value and geometry (Table 1), assuming a flat asteroid spectral response (see Section 5.1). The uncertainties in the relative reflectance values are of the order $30-40 \%$. 
TABLE 4

Corrected IRAC channel 3 and 4 fluxes

\begin{tabular}{lllllllll}
\hline Asteroid & $\begin{array}{c}\text { IRAC 3 } \\
5.731 \mu \mathrm{m} \\
(\mathrm{mJy})\end{array}$ & $\begin{array}{c}\text { Err. 3 } \\
(\mathrm{mJy})\end{array}$ & $\begin{array}{c}\text { IRAC 3 } \\
5.731 \mu \mathrm{m} \\
\left(10^{-17}\right. \\
\left.\mathrm{Wm}^{-2} \mu \mathrm{m}^{-1}\right)\end{array}$ & $\begin{array}{c}\text { Err. 3 } \\
\left(10^{-17}\right. \\
\left.\mathrm{Wm}^{-2} \mu \mathrm{m}^{-1}\right)\end{array}$ & $\begin{array}{c}\text { IRAC 4 } \\
(\mathrm{mJy})\end{array}$ & $\begin{array}{c}\text { Err. 4 } \\
(\mathrm{mJy})\end{array}$ & $\begin{array}{c}\text { IRAC 4.872 } \mu \mathrm{m} \\
\left(10^{-17}\right. \\
\left.\mathrm{Wm}^{-2} \mu \mathrm{m}^{-1}\right)\end{array}$ & $\begin{array}{c}\text { Err. 4 } \\
\left(10^{-17}\right. \\
\left.\mathrm{Wm}^{-2} \mu \mathrm{m}^{-1}\right)\end{array}$ \\
\hline 832 & 1.359 & 0.059 & 12.41 & 0.54 & 13.16 & 0.11 & 63.68 & 0.55 \\
10783 & 0.639 & 0.030 & 5.83 & 0.27 & 4.83 & 0.06 & 23.37 & 0.27 \\
11728 & 0.185 & 0.011 & 1.686 & 0.104 & 1.590 & 0.017 & 7.693 & 0.084 \\
16706 & 0.162 & 0.008 & 1.477 & 0.071 & 1.362 & 0.022 & 6.590 & 0.105 \\
28271 & 0.113 & 0.007 & 1.035 & 0.064 & 1.097 & 0.012 & 5.306 & 0.060 \\
33143 & 0.0654 & 0.0040 & 0.597 & 0.037 & 0.726 & 0.007 & 3.510 & 0.035 \\
34312 & 0.214 & 0.010 & 1.957 & 0.088 & 1.635 & 0.011 & 7.908 & 0.053 \\
40921 & 0.0377 & 0.0035 & 0.344 & 0.032 & 0.400 & 0.008 & 1.934 & 0.041 \\
41307 & 0.0344 & 0.0035 & 0.314 & 0.032 & 0.313 & 0.003 & 1.512 & 0.016 \\
43032 & - & - & & & - & - & & \\
55124 & 0.100 & 0.007 & 0.912 & 0.065 & 0.762 & 0.015 & 3.685 & 0.074 \\
55434 & 0.0756 & 0.0060 & 0.690 & 0.055 & 0.573 & 0.005 & 2.771 & 0.023 \\
71003 & 0.0458 & 0.0053 & 0.418 & 0.048 & 0.520 & 0.007 & 2.515 & 0.033 \\
75176 & 0.0091 & 0.0046 & 0.083 & 0.042 & 0.147 & 0.008 & 0.711 & 0.036 \\
76019 & 0.0282 & 0.0118 & 0.257 & 0.108 & 0.218 & 0.023 & 1.053 & 0.113 \\
76686 & 0.0365 & 0.0041 & 0.333 & 0.037 & 0.445 & 0.010 & 2.151 & 0.046 \\
93690 & 0.0685 & 0.0051 & 0.625 & 0.047 & 0.497 & 0.030 & 2.403 & 0.144 \\
\hline
\end{tabular}

Note: The IRAC channel 3 and 4 fluxes listed here refer to the color-corrected thermal component (see Section 2). 
TABLE 5

Color-corrected IRS PUI fluxes

\begin{tabular}{|c|c|c|c|c|c|c|c|c|}
\hline Asteroid & $\begin{array}{c}\text { PUI } \\
15.8 \mu \mathrm{m} \\
(\mathrm{mJy})\end{array}$ & $\begin{array}{l}\text { Err. } \\
(\mathrm{mJy})\end{array}$ & $\begin{array}{c}\text { PUI } \\
15.8 \mu \mathrm{m} \\
\left(10^{-17}\right. \\
\left.\mathrm{Wm}^{-2} \mu \mathrm{m}^{-1}\right)\end{array}$ & $\begin{array}{c}\text { Err. } \\
\left(10^{-17}\right. \\
\left.\mathrm{Wm}^{-2} \mu \mathrm{m}^{-1}\right)\end{array}$ & $\begin{array}{c}\text { PUI } \\
22.3 \mu \mathrm{m} \\
(\mathrm{mJy})\end{array}$ & $\begin{array}{c}\text { Err. } \\
(\mathrm{mJy})\end{array}$ & $\begin{array}{c}\text { PUI } \\
22.3 \mu \mathrm{m} \\
\left(10^{-17}\right. \\
\left.\mathrm{Wm}^{-2} \mu \mathrm{m}^{-1}\right)\end{array}$ & $\begin{array}{c}\text { Err. } \\
\left(10^{-17}\right. \\
\left.\mathrm{Wm}^{-2} \mu \mathrm{m}^{-1}\right)\end{array}$ \\
\hline 832 & 144.5 & 0.8 & 173.5 & 0.9 & 187.2 & 1.6 & 112.8 & 1.0 \\
\hline 10783 & 23.50 & 0.58 & 28.22 & 0.70 & 27.15 & 0.26 & 16.40 & 0.16 \\
\hline 11728 & 13.33 & 0.16 & 15.97 & 0.19 & 16.10 & 0.22 & 9.70 & 0.13 \\
\hline 16706 & 10.02 & 0.38 & 12.01 & 0.46 & 12.71 & 0.18 & 7.66 & 0.11 \\
\hline 28271 & 6.81 & 0.14 & 8.18 & 0.17 & 7.65 & 0.19 & 4.61 & 0.11 \\
\hline 33143 & 6.60 & 0.29 & 7.93 & 0.35 & 8.83 & 0.22 & 5.32 & 0.13 \\
\hline 34312 & 11.53 & 0.38 & 13.81 & 0.46 & 13.03 & 0.31 & 7.84 & 0.19 \\
\hline 40921 & 4.96 & 0.16 & 5.96 & 0.19 & 6.69 & 0.14 & 4.03 & 0.08 \\
\hline 41307 & 2.69 & 0.10 & 3.23 & 0.12 & 3.33 & 0.10 & 2.01 & 0.06 \\
\hline 43032 & 1.82 & 0.39 & 2.19 & 0.47 & 2.66 & 0.37 & 1.60 & 0.22 \\
\hline 55124 & 5.66 & 0.10 & 6.80 & 0.12 & 6.79 & 0.11 & 4.09 & 0.07 \\
\hline 55434 & 4.37 & 0.09 & 5.25 & 0.11 & 5.19 & 0.10 & 3.13 & 0.06 \\
\hline 71003 & 2.82 & 0.13 & 3.39 & 0.16 & 3.33 & 0.20 & 2.01 & 0.12 \\
\hline 75176 & 1.16 & 0.09 & 1.39 & 0.11 & 1.22 & 0.10 & 0.735 & 0.063 \\
\hline 76019 & 2.06 & 0.08 & 2.47 & 0.10 & 2.55 & 0.09 & 1.537 & 0.054 \\
\hline 76686 & 3.01 & 0.13 & 3.61 & 0.15 & 3.59 & 0.13 & 2.16 & 0.08 \\
\hline 93690 & 3.08 & 0.15 & 3.70 & 0.18 & 3.44 & 0.14 & 2.07 & 0.08 \\
\hline
\end{tabular}


TABLE 6

Separate IRAC and PUI diameters and albedos (mean $\eta$ )

\begin{tabular}{lcccccc}
\hline Asteroid & $\begin{array}{c}D_{\text {irac }} \\
(\mathrm{km})\end{array}$ & $\begin{array}{c}D_{\text {pui }} \\
(\mathrm{km})\end{array}$ & $p_{\text {v irac }}$ & $p_{\text {v pui }}$ & $\eta_{\text {mean }}$ & $\begin{array}{c}\left(D_{\text {irac }}-D_{\text {pui }}\right) \\
D_{\text {irac }}\end{array}$ \\
\hline 832 & 17.73 & 17.49 & 0.19 & 0.19 & 1.00 & 1.35 \\
10783 & 5.56 & 5.37 & 0.16 & 0.17 & 0.82 & 3.42 \\
11728 & 4.68 & 4.49 & 0.27 & 0.29 & 0.86 & 4.06 \\
16706 & 3.48 & 3.22 & 0.16 & 0.19 & 1.13 & 7.47 \\
28271 & 3.39 & 3.34 & 0.29 & 0.30 & 0.80 & 1.47 \\
33143 & 3.68 & 3.29 & 0.19 & 0.24 & 1.26 & 10.6 \\
34312 & 3.03 & 3.05 & 0.21 & 0.21 & 0.80 & -0.66 \\
40921 & 3.41 & 3.36 & 0.18 & 0.19 & 1.14 & 1.47 \\
41307 & 2.44 & 2.42 & 0.16 & 0.16 & 0.90 & 0.82 \\
43032 & - & - & - & - & - & - \\
55124 & 2.73 & 2.51 & 0.16 & 0.19 & 0.90 & 8.06 \\
55434 & 2.05 & 2.45 & 0.24 & 0.17 & 0.78 & -19.5 \\
71003 & 2.85 & 2.51 & 0.18 & 0.23 & 0.98 & 11.9 \\
75176 & 2.01 & 1.72 & 0.11 & 0.15 & 1.24 & 14.4 \\
76019 & 1.40 & 1.68 & 0.33 & 0.23 & 0.83 & -20.0 \\
76686 & 2.78 & 2.82 & 0.19 & 0.18 & 1.12 & -1.44 \\
93690 & 1.55 & 1.85 & 0.42 & 0.30 & 0.69 & -19.35 \\
\hline
\end{tabular}




\section{TABLE 7}

Combined IRAC and IRS PUI diameters, albedos, and $\eta$ values

\begin{tabular}{lrcc}
\hline \multicolumn{1}{c}{ Asteroid } & $D(\mathrm{~km})$ & $p_{\mathrm{v}}$ & $\eta$ \\
\hline 832 & $17.26 \pm 1.73$ & $0.20 \pm 0.07$ & $0.97 \pm 0.01$ \\
10783 & $5.23 \pm 0.52$ & $0.18 \pm 0.07$ & $0.77 \pm 0.01$ \\
11728 & $4.29 \pm 0.43$ & $0.32 \pm 0.12$ & $0.77 \pm 0.01$ \\
16706 & $3.07 \pm 0.31$ & $0.21 \pm 0.08$ & $0.99 \pm 0.02$ \\
28271 & $3.30 \pm 0.33$ & $0.31 \pm 0.11$ & $0.78 \pm 0.02$ \\
33143 & $3.06 \pm 0.31$ & $0.27 \pm 0.10$ & $1.03 \pm 0.02$ \\
34312 & $3.09 \pm 0.31$ & $0.20 \pm 0.07$ & $0.82 \pm 0.02$ \\
40921 & $3.33 \pm 0.33$ & $0.19 \pm 0.07$ & $1.11 \pm 0.02$ \\
41307 & $2.42 \pm 0.24$ & $0.16 \pm 0.06$ & $0.89 \pm 0.02$ \\
43032 & $(2.52)$ & $(0.40)$ & $(1.23)$ \\
55124 & $2.35 \pm 0.24$ & $0.22 \pm 0.08$ & $0.76 \pm 0.02$ \\
55434 & $2.88 \pm 0.29$ & $0.12 \pm 0.04$ & $1.15 \pm 0.02$ \\
71003 & $2.29 \pm 0.23$ & $0.28 \pm 0.10$ & $0.76 \pm 0.03$ \\
75176 & $1.52 \pm 0.15$ & $0.19 \pm 0.07$ & $0.93 \pm 0.06$ \\
76019 & $1.94 \pm 0.19$ & $0.17 \pm 0.06$ & $1.19 \pm 0.10$ \\
76686 & $2.86 \pm 0.29$ & $0.18 \pm 0.07$ & $1.16 \pm 0.04$ \\
93690 & $2.10 \pm 0.21$ & $0.23 \pm 0.09$ & $0.99 \pm 0.04$ \\
\hline
\end{tabular}

Note: Due to the relatively large flux uncertainties and the absence of IRAC data in the case of 43032, the results given here for this object should be treated with caution and are not included in further analysis in this work. 


\section{Figure Captions}

Figure 1. Representative plots of NEATM fits to the $5.7 \mu \mathrm{m}, 7.9 \mu \mathrm{m}, 15.8 \mu \mathrm{m}$, and $22.3 \mu \mathrm{m}$ fluxes listed in Tables 4 and 5. The IRS PUI fluxes $(15.8 \mu \mathrm{m}$, and $22.3 \mu \mathrm{m})$ have been normalized to the geometry of the IRAC fluxes by adjusting them for the slightly different heliocentric and Spitzer-centric distances and solar phase angles using the NEATM. Error bars are $1 \sigma$.

Figure 2. Histogram of the albedo distribution of the Karin targets. The horizontal dashed line is a representative $1 \sigma$ error bar from our analysis.

Figure 3. Albedo versus diameter for (a) the observed Karin cluster asteroids and (b) for a set of S- and Q-type near-Earth asteroids (plot adapted from Harris, 2006, with the addition of data from the compilation of Wolters et al., 2008; the horizontal line at $p_{\mathrm{v}}=0.215$ corresponds to the mean albedo of the observed Karin asteroids). In contrast to the near-Earth asteroid albedos the Karin cluster albedos show no dependence on diameter.

Figure 4. The derived diameters of the observed Karin cluster asteroids versus the NEATM modeling parameter $\eta$. There is no obvious trend of asteroid size with $\eta$.

Figure 5. The derived albedos of the observed Karin cluster asteroids versus the NEATM modeling parameter $\eta$. There is a possible trend of decreasing albedo with increasing $\eta$.

Figure 6. Histogram of the $\eta$-value distribution of the Karin targets. Values of $\eta$ consistent with thermal inertia $\Gamma=0,30,200 \mathrm{Jm}^{-2} \mathrm{~s}^{-0.5} \mathrm{~K}^{-1}$ for a rotation period of $20 \mathrm{~h}$ are indicated; these were calculated using a smooth-sphere thermophysical model and represent maximum values for a sample of objects with random spin-axis orientations and rough (cratered) surfaces. The $\eta$-value distribution falls far short of the expected maximum for $\Gamma=200 \mathrm{Jm}^{-2} \mathrm{~s}^{-0.5} \mathrm{~K}^{-1}$, 
suggesting the thermal inertia values of the Karin cluster asteroids are much less than those of comparably-sized near-Earth asteroids. 


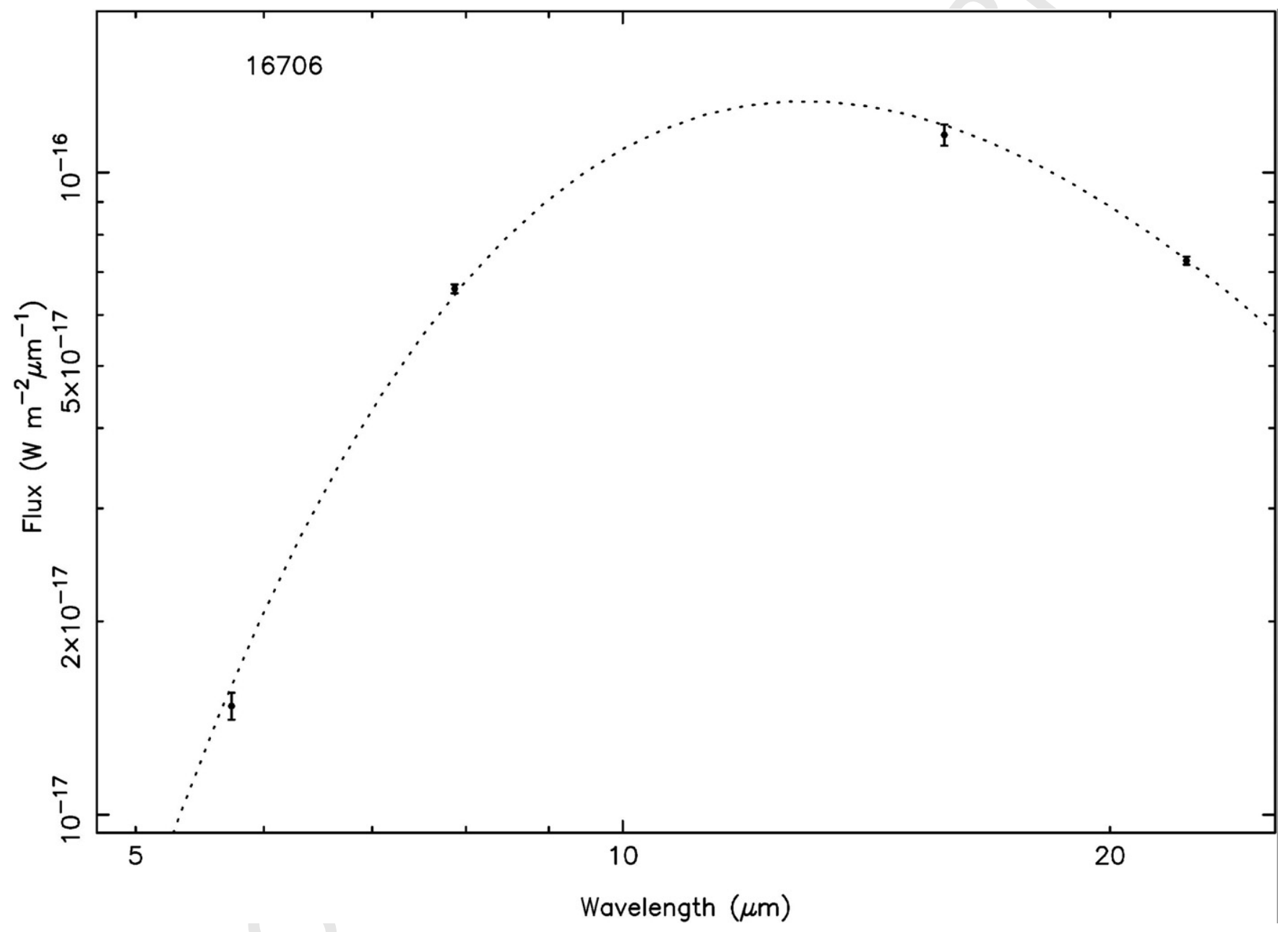




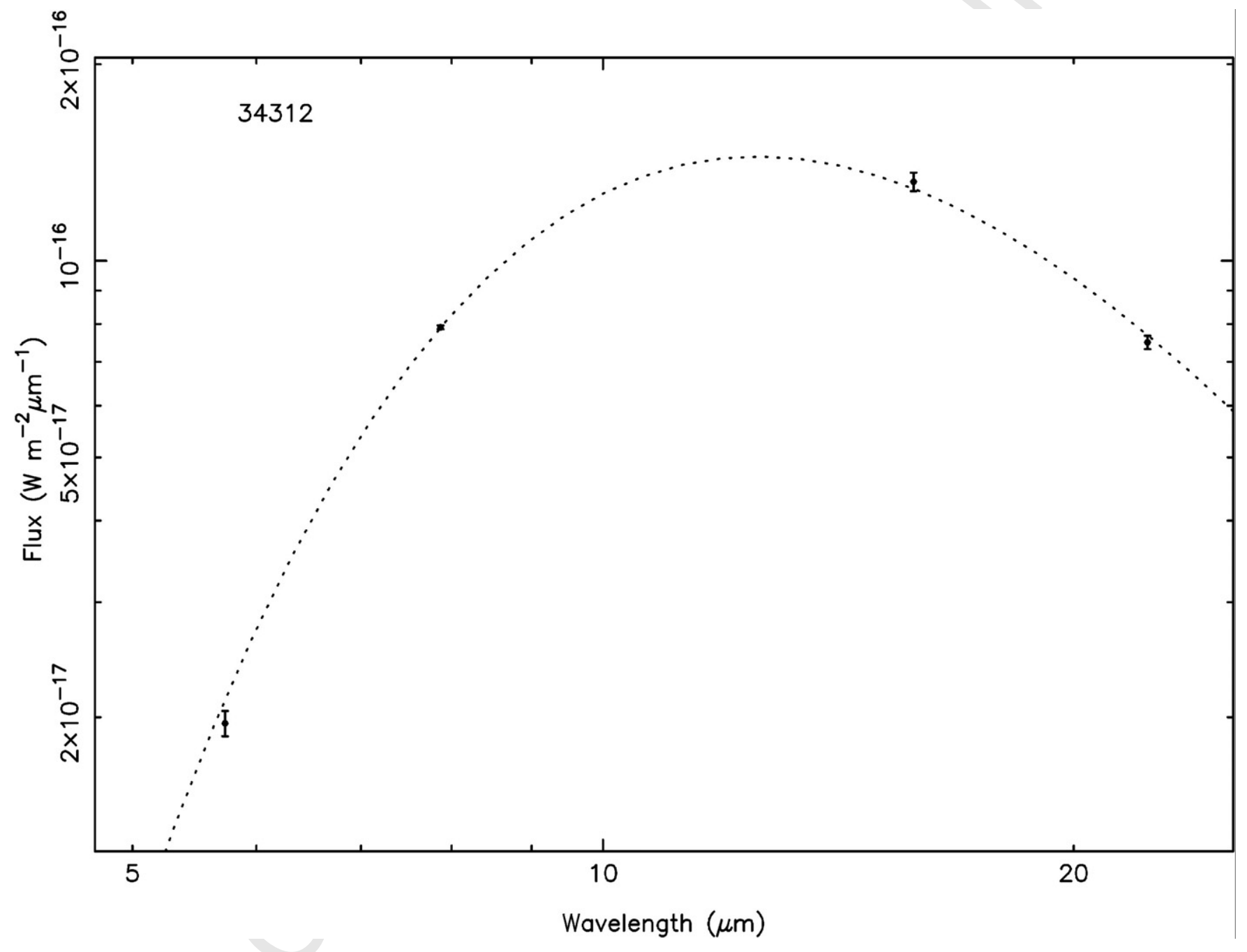




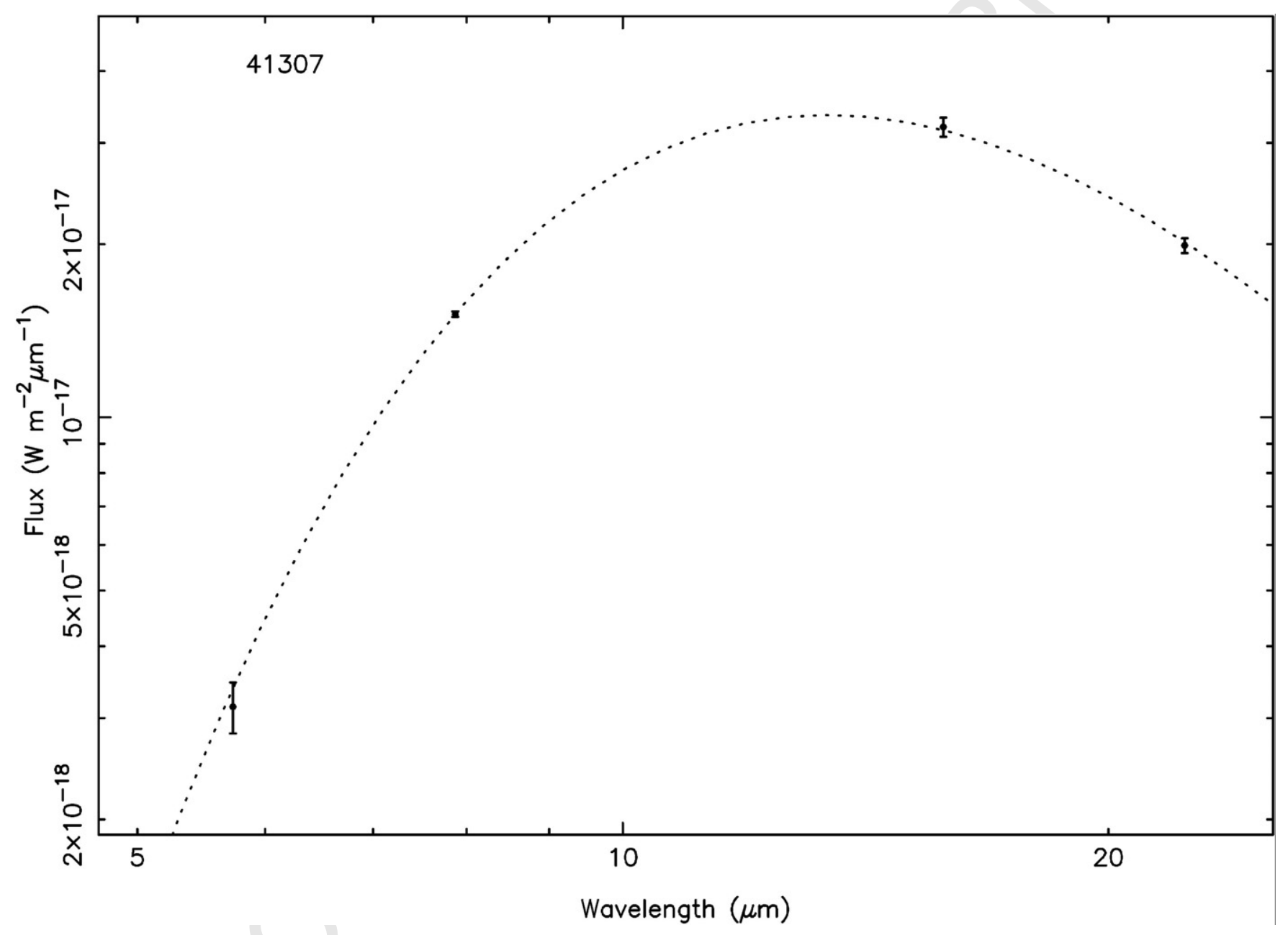




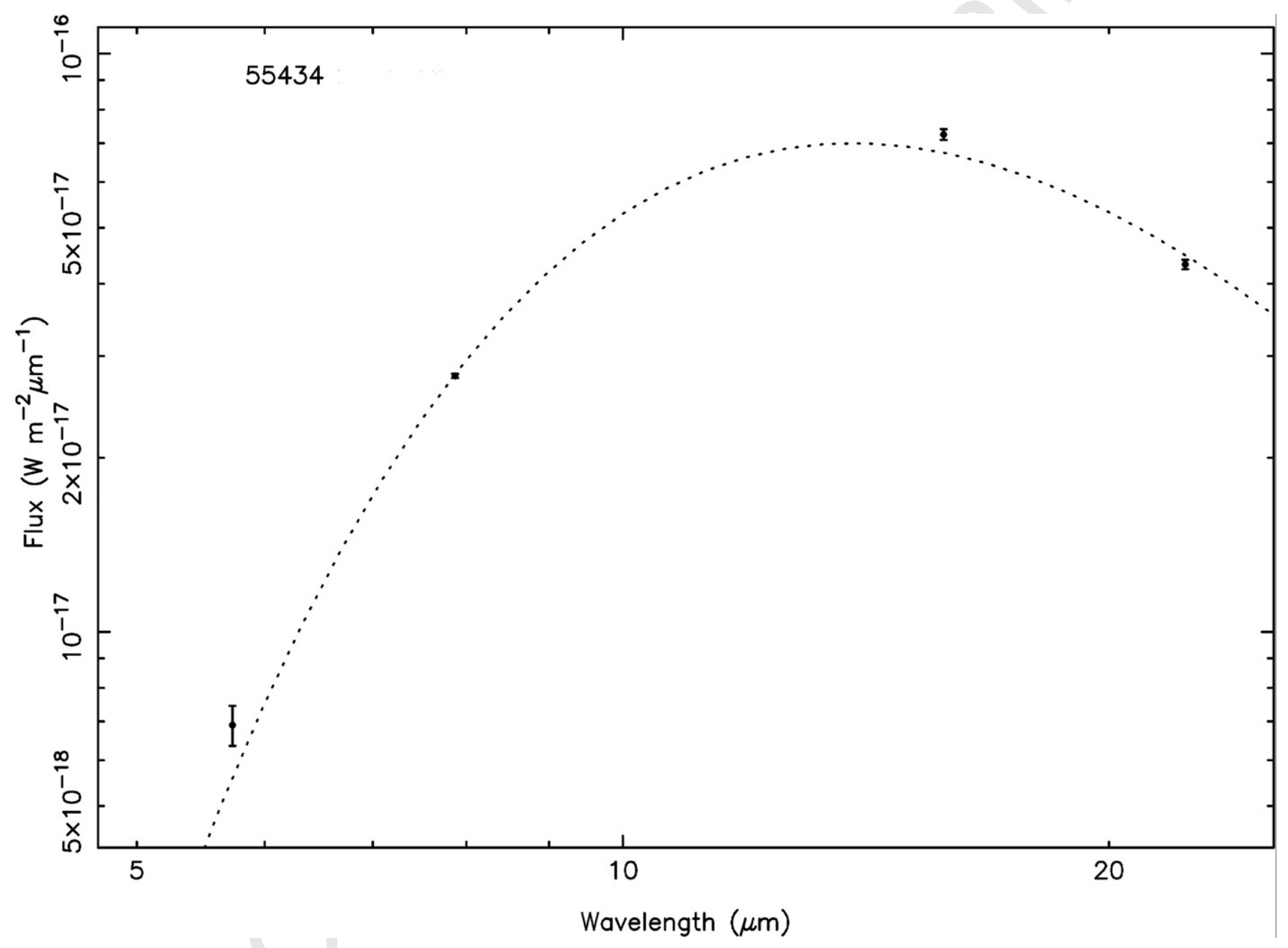




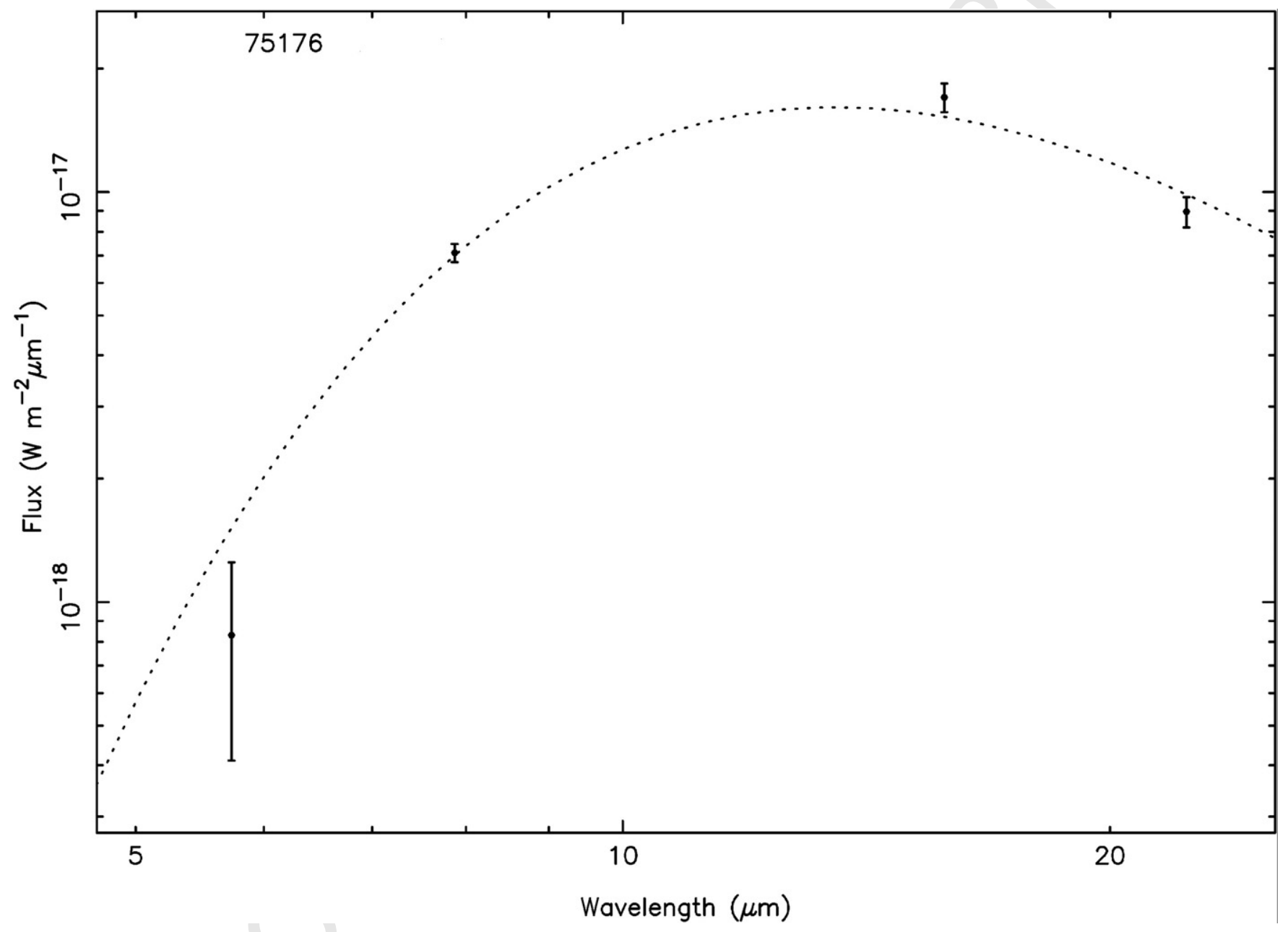




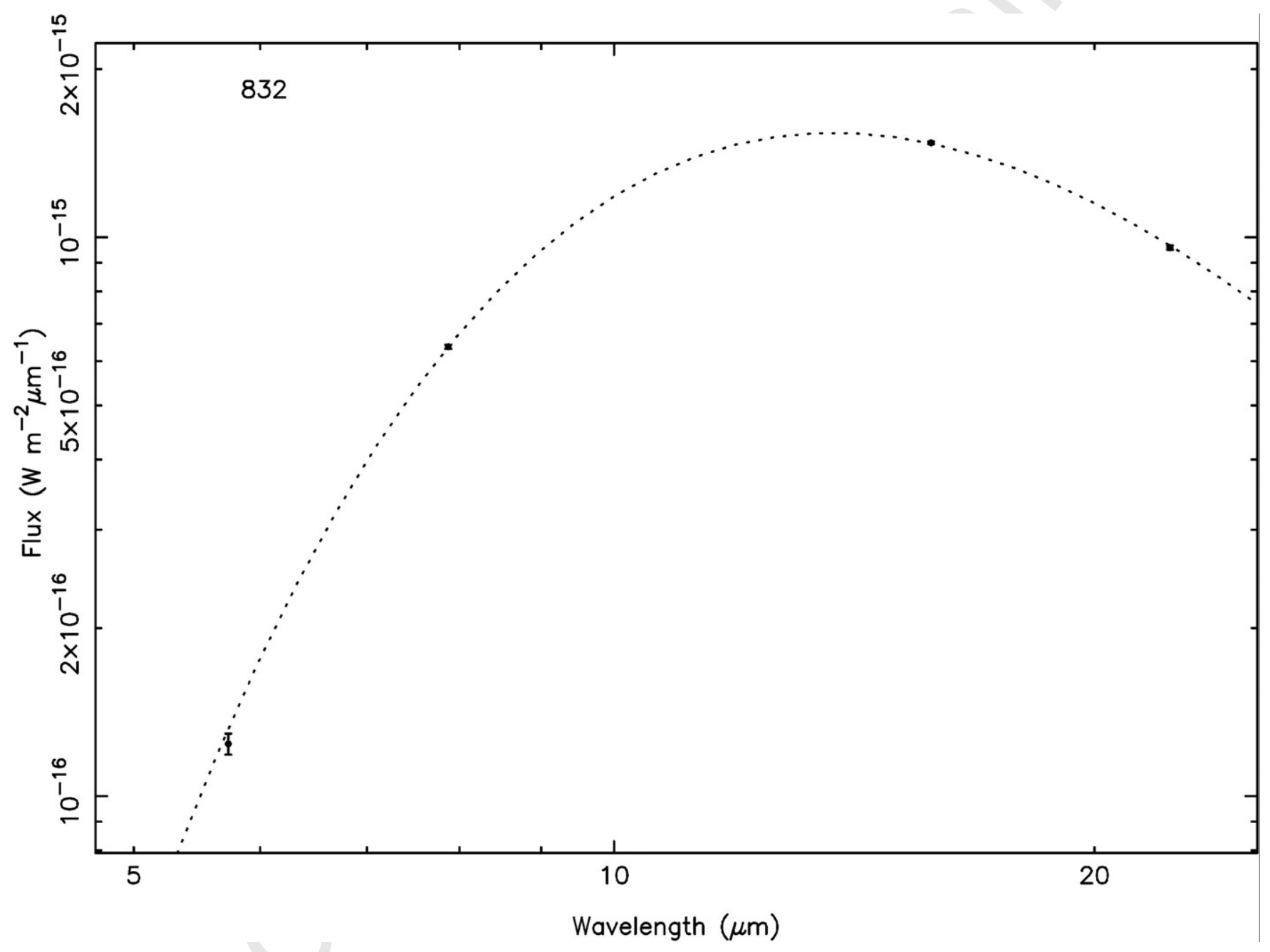




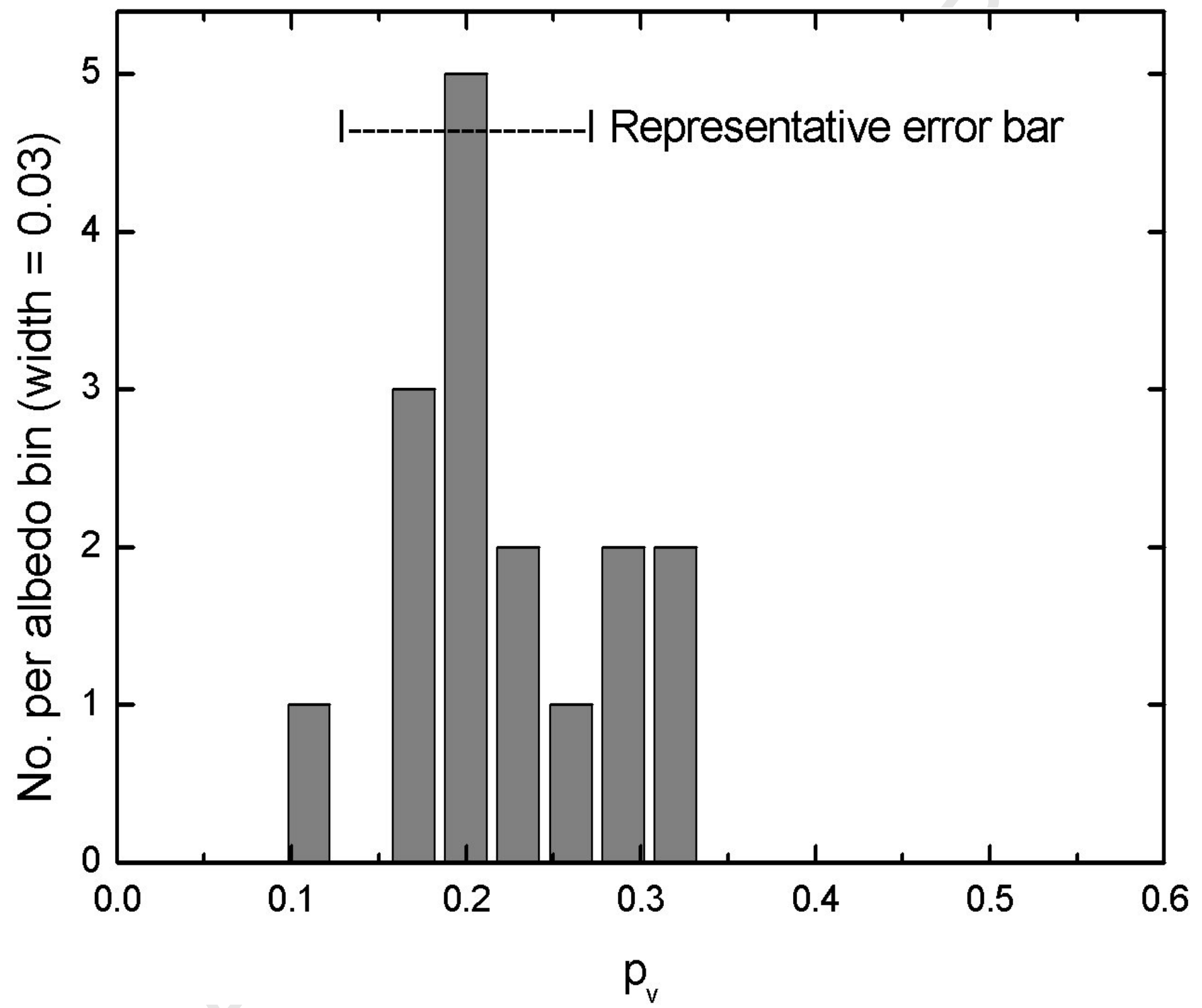




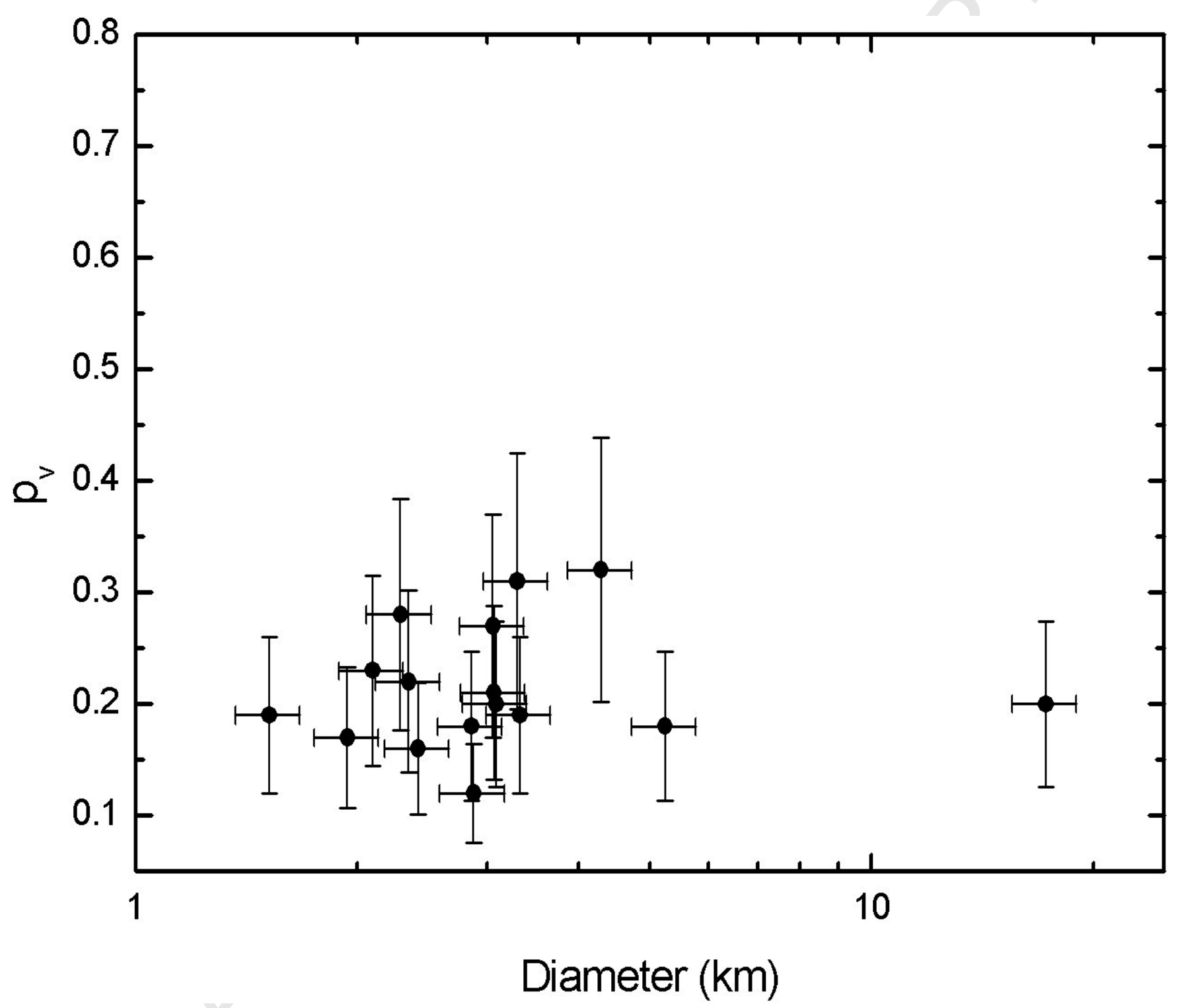




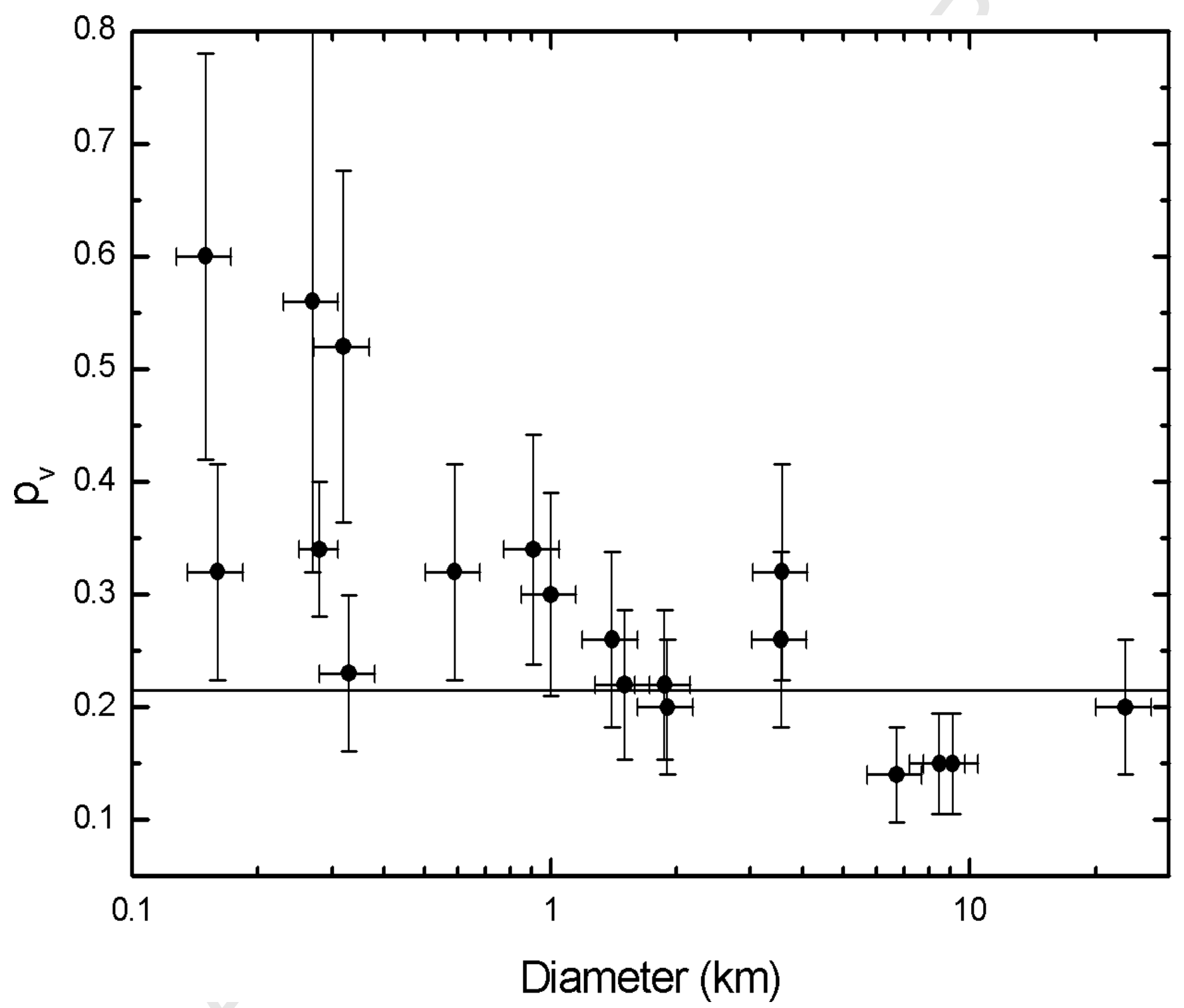




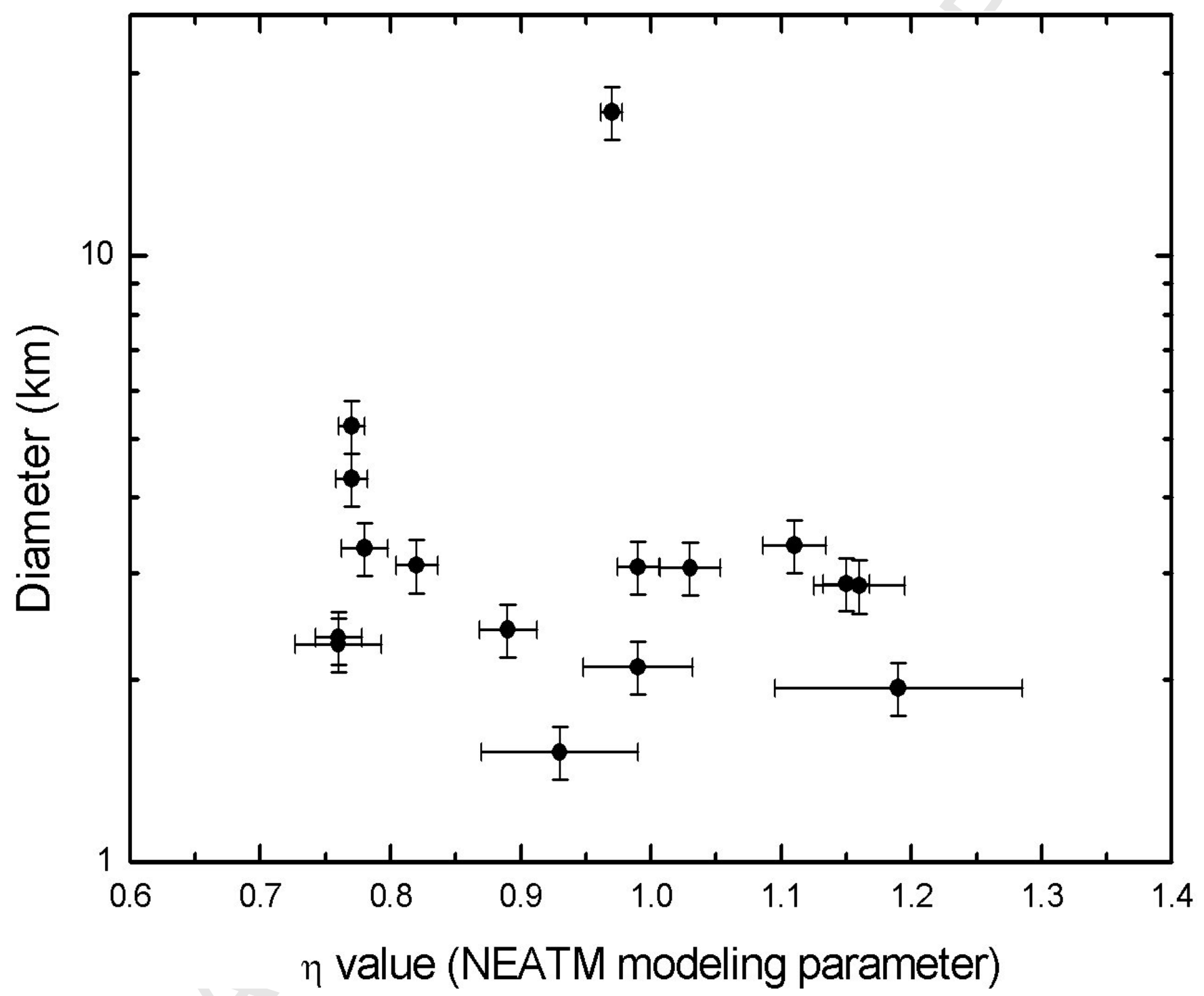




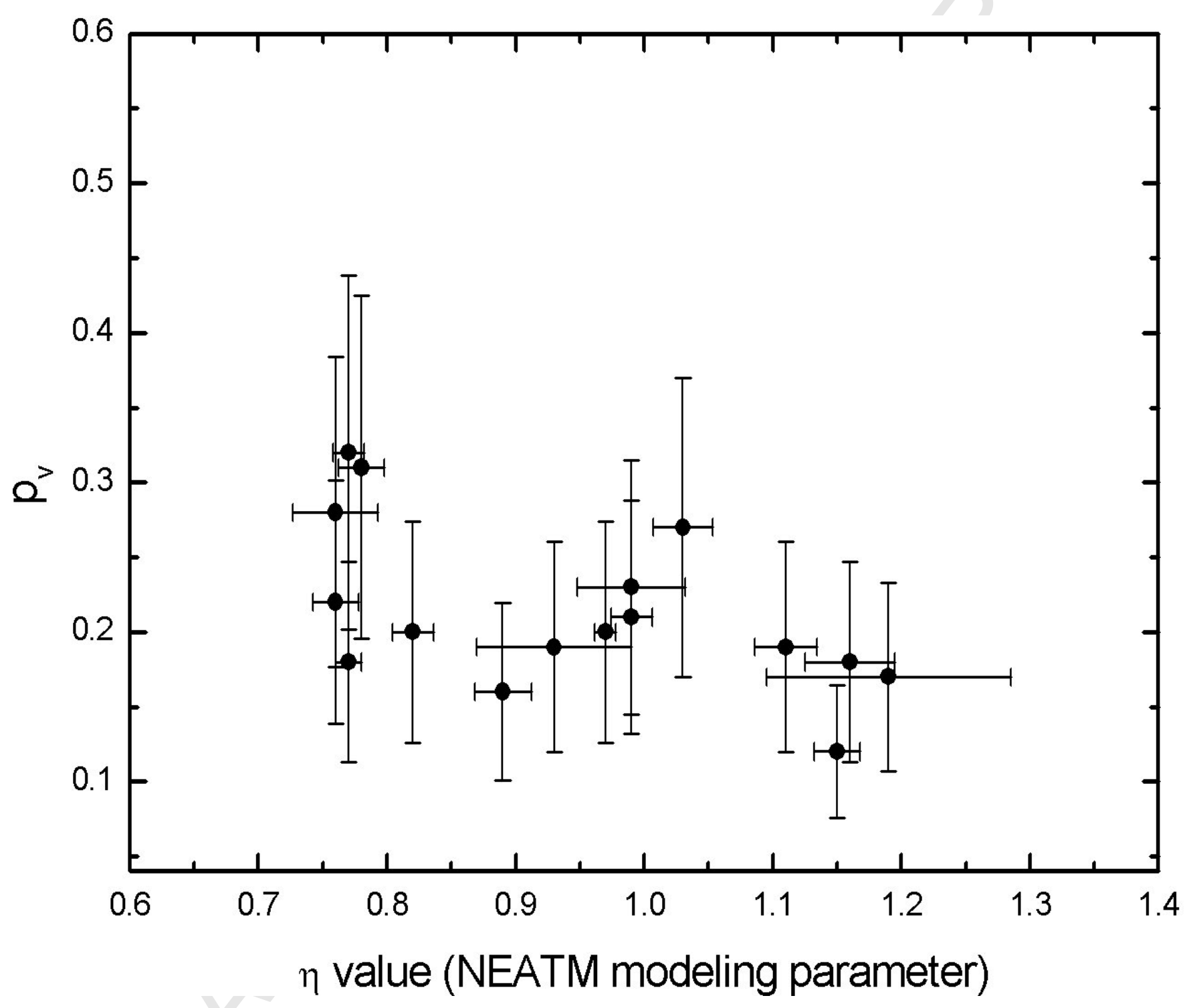




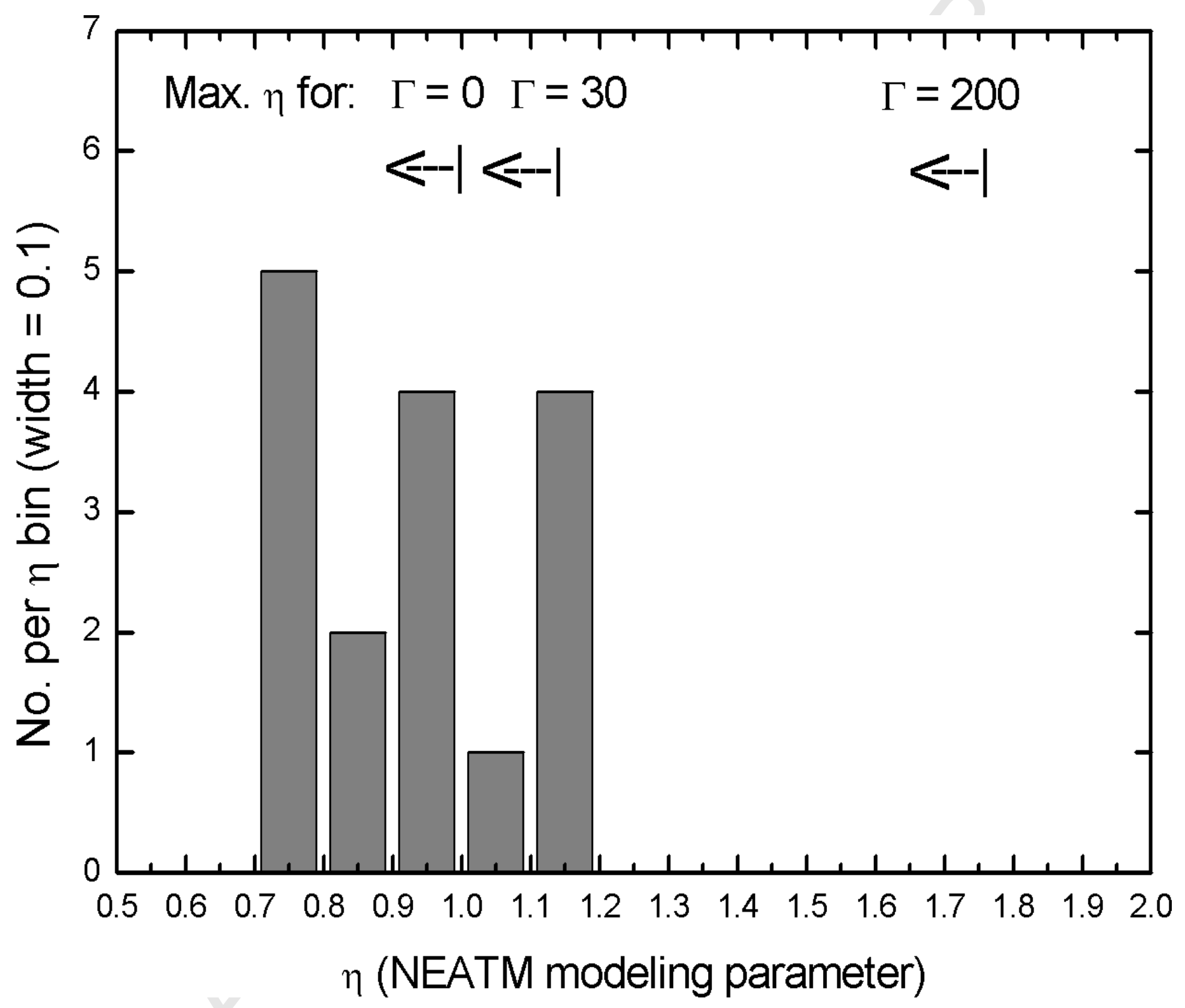

